\title{
A Novel Data Collection Method with Recharge Plan for Rechargeable Wireless Sensor Networks
}

\author{
Chi-Chang Chen (1) \\ Department of Information Engineering, I-Shou University, Kaohsiung City 84001, Taiwan \\ Correspondence should be addressed to Chi-Chang Chen; ccchen@isu.edu.tw
}

Received 10 December 2017; Revised 26 February 2018; Accepted 28 February 2018; Published 2 April 2018

Academic Editor: Alessandra De Paola

Copyright (c) 2018 Chi-Chang Chen. This is an open access article distributed under the Creative Commons Attribution License, which permits unrestricted use, distribution, and reproduction in any medium, provided the original work is properly cited.

\begin{abstract}
Because of recent advances in wireless power transfer technologies, several key topics of wireless sensor networks (WSNs) need to be revisited. Traditional data collection methods for WSNs typically consider the balance of power consumption among sensors as a critical design criterion for avoiding uneven power depletion in the networks. I propose a solution for the uneven power consumption problem of data collection over WSNs. I designed a node-Gosper island-based scalable hierarchical cluster transmission method in conjunction with a wireless recharge plan for data collection over rechargeable WSNs. For the recharge plan, I used mobile wireless chargers to visit and recharge the batteries of sensors located on different levels of node-Gosper curves with various frequencies. The duration of each recharging process for each layer of sensors was calculated to verify the feasibility of the proposed recharge plan. The simulation results indicate that my proposed data collection method outperforms several wellknown data collection methods in terms of energy consumption and that my proposed recharge plan is more efficient than previous approaches in terms of charge path length, number of alive nodes, and traveling efficiency.
\end{abstract}

\section{Introduction}

A wireless sensor network (WSN) is a wireless communication network consisting of numerous automated devices that use sensors to cooperate in monitoring the physical or environmental conditions of designated locations, such as temperature, sound, vibration, pressure, movement, and contaminants. The development of WSNs originated in battlefield monitoring and other military applications. WSNs are used in many application areas such as environmental and ecological monitoring, health monitoring, home automation, and traffic control.

In recent years, the number of studies on WSNs has increased. In such studies, the method of extending the lifetime of WSNs is always the key topic considered for designing protocols or algorithms for WSNs. In the literature, strategies to extend the lifetime of WSNs can be divided into two categories. The first category comprises strategies to decrease the energy consumption of WSNs to the greatest extent possible through methods such as designing low power hardware architectures, using power-saving communication technologies, installing low complexity operating software, developing various types of energy-saving data collection protocols or positioning algorithms, and using mobile anchor nodes to participate in the operation of the sensor networks. Although previous efforts have provided several effective energy-saving methods, the ability to extend the lifetime of WSNs is still limited by the battery power configured in the sensors.

The second category comprises energy harvesting techniques that use surrounding environmental natural energies such as sunlight, terrestrial heat, wind, vibration, and bioenergies. These techniques result in WSNs not using batteries as their only source of energy. Presently, the most successful method in this category is the use of solar energy. However, the acquisition of solar energy is uncertain because of environmental and weather-related factors. Thus, guaranteeing continuous and stable energy supplies for WSNs is difficult.

Over the preceding few years, wireless charging technologies have undergone rapid development, especially regarding their application in mobile phones. Many experts believe that wireless charging will become a basic function of the nextgeneration of mobile phones. Wireless charging technologies can be formally divided into two categories according to their 


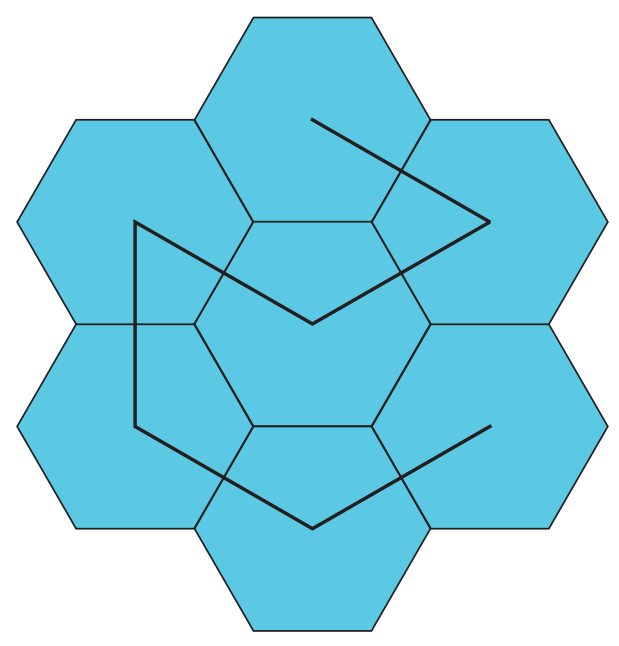

(a)

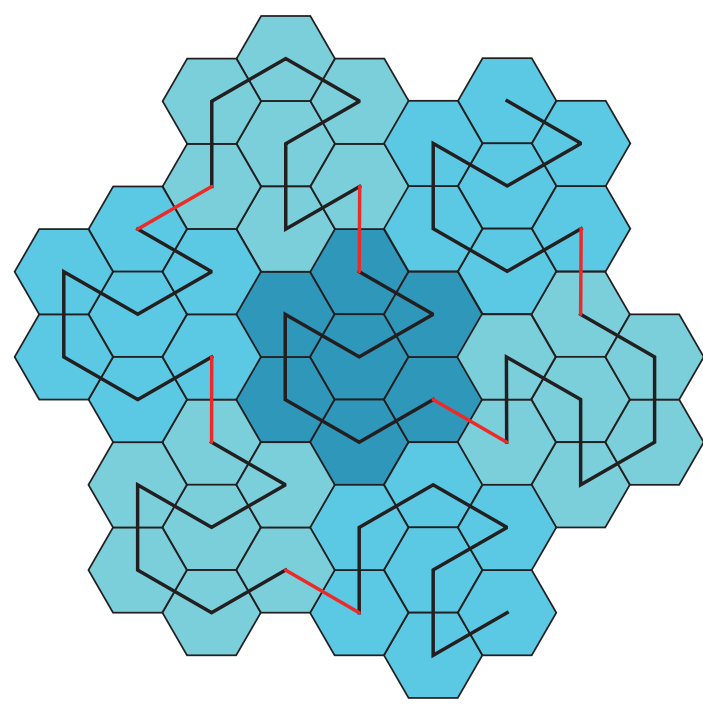

(b)

FIGURE 1: (a) Level-1 node-Gosper island and corresponding node-Gosper curve. (b) Level-2 node-Gosper island and corresponding nodeGosper curve.

transmission range: far-field systems and near-field systems [1]. Near-filed wireless power transfer systems, according to their transfer distance, can be further divided into short range near-field power transfer systems and midrange near-field power transfer systems [1].

Midrange near-field power transfer systems mainly use the electromagnetic resonance power transmission principle, which enables a coil to simultaneously charge several devices at distances of $2 \mathrm{~m}$ or more; however, the devices' performance decreases with distance. The current industry standard for the electromagnetic resonance power transmission technique is maintained by the Alliance for Wireless Power (A4WP; the A4WP merged with the Power Matters Alliance in 2016 to form the AirFuel Alliance). The WiTricity Corporation is the most major company producing devices that support this technology.

Short range near-field power transfer systems mainly apply the electromagnetic induction power transmission principle, in which a coil can charge only one device at a time and the charge distance is generally less than $4 \mathrm{~cm}$. Therefore, the charging source is typically in direct contact with the charged device. The widely used industry standard for this technology is Qi, which is maintained by the Wireless Power Consortium.

In this work, I assume two types of wireless chargers are used. Each charger is assumed to be a robot car equipped with a wireless communication device, global position system (GPS), and charge devices with the capability to wirelessly charge sensors by using electromagnetic resonance power transmission technology or radio frequency power transmission technology depending on the type of sensors to be charged.

A node-Gosper curve is a space-filling curve of fractal geometry generated by the continuous replacement of each vertex with the basic seven-segment curve. The resultant filled space is a node-Gosper island [2, 3].
Figures 1(a) and 1(b) show level-1 and level-2 node-Gosper islands, respectively, with their corresponding node-Gosper curves.

The remainder of this paper is organized as follows. Section 2 introduces the background of wireless charging technology as well as definitions and properties of nodeGosper islands and node-Gosper curves. Section 3 presents several classic data collection methods for WSNs and recent studies of recharge methods for RWSNs. Section 4 presents my proposed data collection method and recharge plan for RWSNs. The performance of my data collection method and recharge plan are simulated and compared with those of other methods in Section 5. Finally, Section 6 concludes this work.

\section{Background}

2.1. Review of Wireless Charging Technology. Wireless charging technology originated approximately 100 years ago with American scientist Nikola Tesla. Tesla succeeded in delivering electricity to a target electrical appliance in the form of wireless energy transmission. He then began working on commercial intercontinental wireless power delivery and built a semifinished product called the Wardenclyffe Tower. However, at the time, no one believed that the wireless transmission of electricity was necessary. Additionally, there were concerns about its security, causing Tesla's plan to fail. The Wardenclyffe Tower was eventually demolished.

In 2007, at the Massachusetts Institute of Technology (MIT), a group of theoretical physicists performed an electromagnetic resonance power transmission of $2 \mathrm{~m}$ to light a $60-\mathrm{W}$ bulb [4], thereby bringing wireless power transmission into a new era. A general block structure of a resonant wireless charging system is illustrated in Figure 2. The structure uses a DC/RF amplifier as a high-efficiency switching amplifier to convert DC voltage into an RF voltage waveform used to drive the source resonator. 


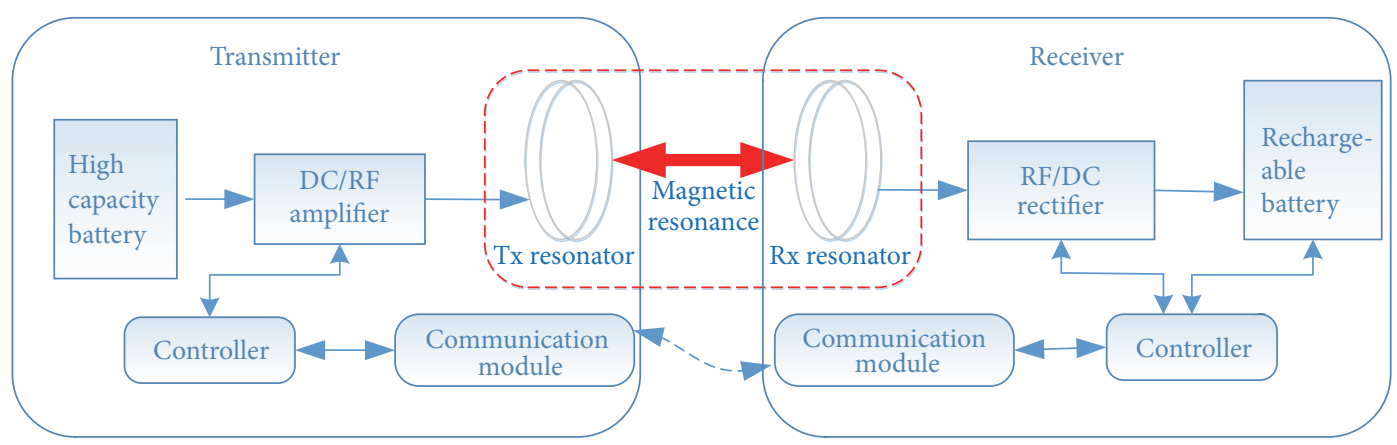

FIGURE 2: General resonant wireless charging system.

TABLE 1: Experimental results of magnetic resonance power transfer efficiency versus distance between source and receiver*.

\begin{tabular}{lcccccc}
\hline Distance $(\mathrm{cm})$ & 100 & 125 & 150 & 175 & 200 & 225 \\
$\eta$ (efficiency) & 0.90 & 0.81 & 0.73 & 0.52 & 0.51 & 0.31 \\
\hline
\end{tabular}

${ }^{*}$ Data were from [4], and the power transferred was able to light up a $60 \mathrm{~W}$ light bulb.

Current near-field wireless charging systems can be classified into two categories [1]:

(1) Midrange charging, one of the representative manufacturers of which is the WiTricity Corporation, an MIT derivative company,

(i) uses electromagnetic resonance power transmission;

(ii) can simultaneously charge a variety of devices;

(iii) can charge at distances of $2 \mathrm{~m}$ or more but the performance decreases with distance; and

(iv) is suitable for home appliance and electric car charging.

(2) Short range charging, one of the major protocols of which is Qi,

(i) uses electromagnetic induction power transmission;

(ii) can only charge one device at a time per coil;

(iii) can charge at distances of less than $4 \mathrm{~cm}$ (the charging source is typically in direct contact with the charged device); and

(iv) is typically used for mobile phone charging.

In a magnetic resonance system, the ratio of two characteristic parameters, namely, resonant frequency $\omega$ and intrinsic loss rate $\Gamma$, of each isolated magnetic resonator defines the quality factor, $Q$, of the resonator, where $Q=$ $\omega / 2 \Gamma$. In a highly resonant wireless power transfer system, the system resonators must have a high quality factor to transfer energy. The efficiency of the energy transfer depends on the characteristic parameters for each resonator and the energy coupling rate, $k$, between them. The efficiency of the power transmission can be maximized by the formula $\eta_{\text {opt }}=$ $U^{2} /\left(1+\sqrt{1+U^{2}}\right)^{2}$, where $U=k \sqrt{Q_{s} Q_{d}}$, and $Q_{s}$ and $Q_{d}$ are quality factors of source resonator and receiver resonator.
By setting up and tuning the appropriate parameters for the magnetic resonance system, the MIT team in [4] obtained the experimental results, shown in Table 1, of magnetic resonance power transfer efficiency $\eta$ versus the distance between source and receiver resonators. For more details regarding the magnetic resonance system of wireless power transfer, please refer to $[4,5]$.

Another type of power transfer technology is to use radio frequency signals. According to the Friis free space equation, the authors in [6] proposed a power transmission formula indicating that power transmission energy and distance are inversely proportional to the square. The formula is expressed as follows:

$$
p_{r}=\frac{G_{s} G_{r} \eta}{L_{p}}\left(\frac{\lambda}{4 \pi(d+\beta)}\right)^{2} p_{0}
$$

where $p_{r}$ and $p_{0}$ are the power of the receiver and the source, respectively; $G_{s}$ and $G_{r}$ are the antenna gain of the source and receiver, respectively; $\eta$ is the correction performance; $L_{p}$ is polarization loss; $d$ is the distance between the source and the receiver; $\lambda$ is the wavelength; and $\beta$ represents the Friis free space correction parameters.

In this work, I assume two types of wireless charge cars are used. Each charge car is a robot car equipped with a wireless communication device, global position system (GPS), and charge devices with the capability to wirelessly charge sensors by using electromagnetic resonance power transmission technology or by radio frequency power transmission technology, depending on the type of sensors to be charged.

2.2. Definitions of Node-Gosper Islands and Node-Gosper Curves. A Gosper curve is a spatial filling curve of fractal geometry that passes through all hexagons that are seamlessly arranged in a spatial region without intersecting [2]. The area filled by the Gosper curve is called a Gosper island. To easily describe the trajectory of the Gosper curve, which 


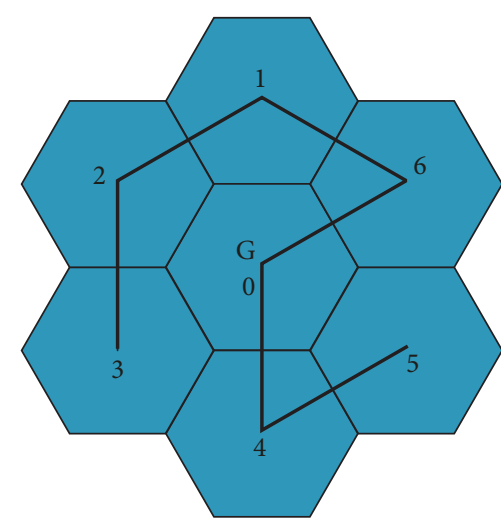

(a)

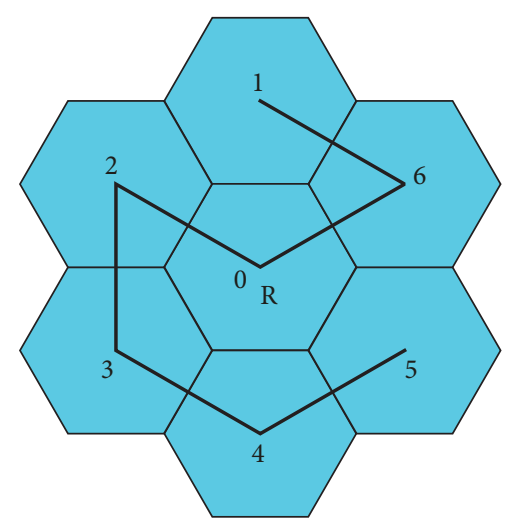

(b)

Figure 3: Level-1 node-Gosper islands with (a) G-type node-Gosper curve and (b) R-type node-Gosper curve.

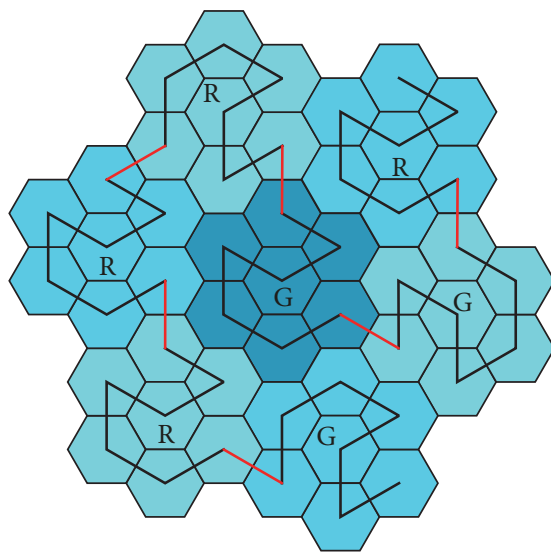

(a)

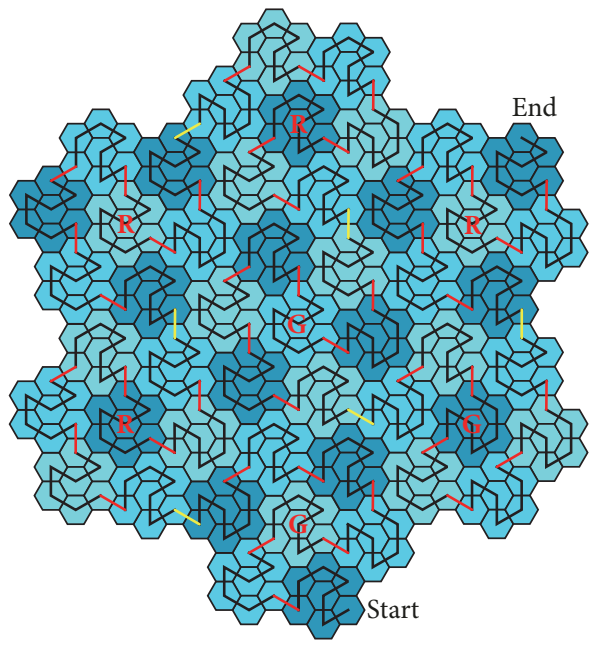

(b)

FIGURE 4: (a) Level-2 node-Gosper island with R-type node-Gosper curve and (b) Level-3 node-Gosper island with R-type node-Gosper curve.

is used as the traveling path of the mobile wireless charger, and define the coordinates of each vertex of the curve, I changed the trajectory of the Gosper curve to pass through the center of each hexagon of the Gosper island. Therefore, in this study, the rule of generating a higher level Gosper curve was changed to replace the vertices rather than the segments of the current level curve with the level-1 Gosper curve. In contrast to the traditional definition of a Gosper curve, this new Gosper curve is called a node-Gosper curve $[2,3]$. Figures 3(a) and 3(b) illustrate two types of level-1 node-Gosper curves, type $\mathrm{G}$ and type $\mathrm{R}$, respectively. The filled area is called a level-1 node-Gosper island. Figures 4(a) and 4(b) depict the level-2 and level-3 node-Gosper islands, respectively.

Originally, the Gosper curve was mainly applied in the design of communication antennas, as in [7, 8], and occasionally in visual image processing, as in [9]. Recently, the node-Gosper curve has been successfully applied to solve the mobile anchor localization problems of WSNs [3].
2.3. Basic Concepts and Properties of Node-Gosper Curves. To describe the loci of node-Gosper curves, this section briefly introduces two addressing methods suitable for identifying hexagons in node-Gosper islands. For more details, please refer to my previous study [3].

(1) Addressing Methods of Node-Gosper Islands. In the literature, a variety of hexagonal addressing methods for identifying hexagons in a hexagonal tessellated plane are described. In the present study, I used two addressing methods, namely, the ring-based addressing scheme and base-7 addressing scheme, described in [3], both of which are suitable for addressing the hexagons in a node-Gosper island.

In the ring-based addressing scheme, the plane tessellated with regular hexagons is considered as comprising concentric hexagonal rings centered at the center of the plane, as illustrated in Figure 5(a). Suppose that the center of the plane is at the origin $(0,0)$ and the plane is tessellated with regular hexagons of length $r$. For simplicity, I assume that $r=1$ in the following discussion. Let the center of the central 


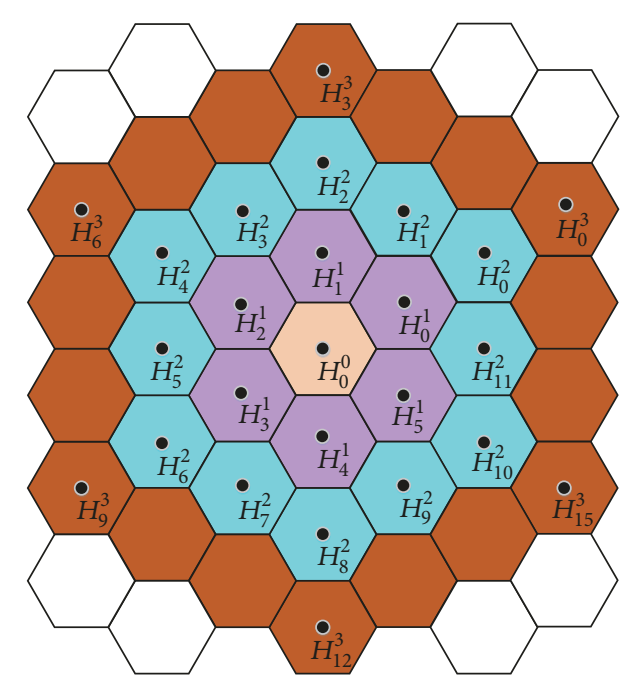

(a)

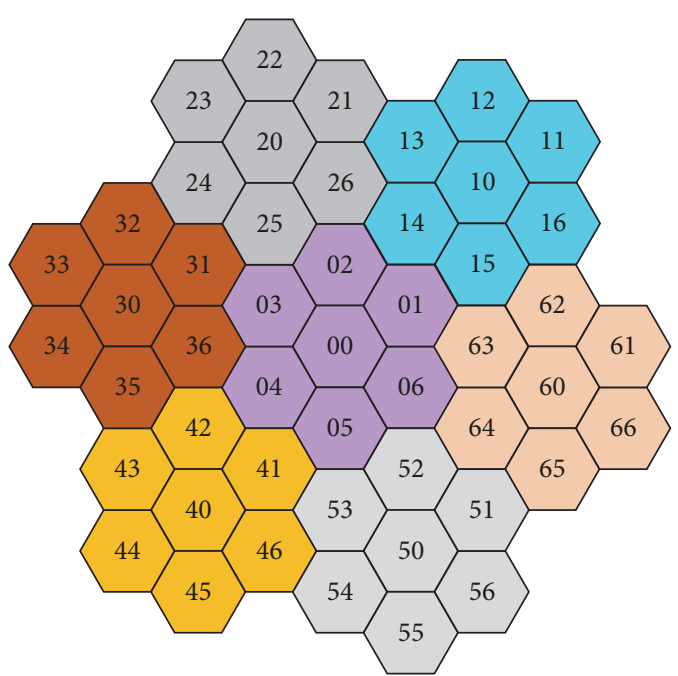

(b)

FIGURE 5: Examples of two addressing methods for hexagons in a hexagonal tessellation plane. (a) Ring-based addressing of the first three rings, and (b) base-7 addressing of the level-2 node-Gosper island.

hexagon be denoted by $H_{0}^{0}$. Thus, $H_{0}^{0}=(0,0)$. The hexagons immediately surrounding the central hexagon constitute the first hexagonal ring. Similarly, the hexagons immediately surrounding the $(n-1)$ th hexagonal ring constitute the $n$th hexagonal ring. The center of the $k$ th hexagon in the $n$th hexagonal ring is denoted as $H_{k}^{n}$. The notations used to represent hexagon centers in the first hexagonal ring are shown in Figure 5(a). Six hexagons exist in the first hexagonal ring. Their centers, in Cartesian coordinates, can be calculated from their polar coordinates as follows:

$$
H_{i}^{1}=\sqrt{3}\left(\cos \left(\frac{\pi}{6}+\frac{i \times \pi}{3}\right), \sin \left(\frac{\pi}{6}+\frac{i \times \pi}{3}\right)\right),
$$

$$
\text { where } i=0,1, \ldots, 5 \text {. }
$$

The center of the $k$ th hexagon in the $n$th hexagonal ring can be represented as the combination of $H_{i}^{1}$ and $H_{i+1}^{1}$, expressed as follows:

$$
\begin{aligned}
& H_{k}^{n}=(n-(k-n \times i)) H_{i}^{1}+(k-n \times i) H_{i+1}^{1}, \\
& \text { where } i=\left\lfloor\frac{k}{n}\right\rfloor .
\end{aligned}
$$

In a level- $n$ node-Gosper island, it is possible to uniquely index every hexagon by using an $n$-digit base- 7 number, where each digit indicates the specific position of a lower level node-Gosper island in the super-level node-Gosper island; 0 indicates the center hexagon, and numerals 1-6 indicate hexagons counted counterclockwise around the center hexagon. This method of indexing each hexagon in a Gosper island is referred to as the base-7 addressing scheme. Figure 5(b) shows the indexes of hexagons in a level-2 nodeGosper island. The Cartesian coordinates of the center point for a hexagon addressed by the base- 7 addressing scheme can be calculated from the linear combination of ring-based addresses, as stated in Theorem 1 . The proof and examples of Theorem 1 are provided in [3].
Theorem 1. Assume that hexagon A in the level-n node-Gosper island has a base-7 address of $d_{n} d_{n-1} \cdots d_{2} d_{1}$. The Cartesian coordinates of the center point of $A$ are expressed as follows:

$$
\operatorname{Center}(A)=\sum_{i=1}^{n} H_{\left(d_{i}-1\right) \times r(i)+r(i-1) \times[(i-1) \bmod 2]}^{r(i)}
$$

where $r(k)$ and $k \geq 1$ represent the rank of the hexagonal ring where the central hexagons of the surrounding level- $(k-1) s u b$ node-Gosper islands are located, and $r(0)=0$.

(2) Path Description of Node-Gosper Curves. Theorem 1 calculates the coordinates of the center of each hexagon in the node-Gosper island. These center points are the vertices of the corresponding node-Gosper curve. In this section, I describe the node-Gosper curve through a sequence of vertices by using the base- 7 addressing scheme. This sequence can be converted to the corresponding sequence of Cartesian coordinates through Theorem 1.

As shown in Figures 3(a) and 3(b), two types of nodeGosper curves exist, namely, type $G$ and type R. The higher levels of node-Gosper curves are constructed by connecting these two types of node-Gosper curves in the lower levels. The two types of node-Gosper curves can be described using the production rules of the L-system defined in [2] as follows:

Type G:

$$
\begin{aligned}
& \mathrm{G} \leftarrow \mathrm{G} r r \uparrow l \mathrm{R} r r \uparrow l \mathrm{R} r \uparrow l l \mathrm{G} r \uparrow l l \mathrm{G} r r \uparrow \\
& l \mathrm{G} r \uparrow l l \mathrm{R} \| \uparrow r r \uparrow r \uparrow l l \uparrow l \uparrow l \uparrow
\end{aligned}
$$

Type R:

$$
\begin{aligned}
& \mathrm{R} \leftarrow \mathrm{G} r r \uparrow l \mathrm{Rr} \uparrow l l \mathrm{R} r r \uparrow l \mathrm{R} r r \uparrow l \mathrm{Gr} \uparrow \\
& l l \mathrm{Gr} r l l \mathrm{R} \| \uparrow r \uparrow r \uparrow r r \uparrow l \uparrow l l \uparrow,
\end{aligned}
$$


where $\{\mathrm{G}, \mathrm{R}\}$ are nonterminal symbols, $\{r, l, \uparrow\}$ are terminal symbols, $r$ and $l$ represent a right-turn of $60^{\circ}$ and left-turn of $60^{\circ}$, respectively, and $\uparrow$ represents moving straight forward.

As an example, Figure 4(a) shows a level-2 node-Gosper island with its corresponding type $\mathrm{R}$ node-Gosper curve, which can be described using the aforementioned production rules as follows:

$$
\begin{aligned}
& \uparrow r r \uparrow r \uparrow l l \uparrow l \uparrow l \uparrow r r \uparrow l \uparrow r \uparrow r \uparrow r r \uparrow l \uparrow l l \uparrow r \\
& \uparrow l l \uparrow r \uparrow r \uparrow r r \uparrow l \uparrow l l \uparrow r r \uparrow l \uparrow r \uparrow r \uparrow r r \uparrow l \uparrow l l \\
& \uparrow r r \uparrow l \uparrow r r \uparrow r \uparrow l l \uparrow l \uparrow l \uparrow r \uparrow l l \uparrow r r \uparrow r \uparrow l l \uparrow l \uparrow \\
& l \uparrow r \uparrow l l \uparrow r \uparrow r \uparrow r r \uparrow l \uparrow l l \uparrow .
\end{aligned}
$$

In this study, I used the base-7 addressing scheme to clearly describe the loci of node-Gosper curves. For example, the path of the type G level-1 node-Gosper curve in Figure 3(a) can be described using the base-7 addressing scheme as $5 \rightarrow 4 \rightarrow 0 \rightarrow 6 \rightarrow 1 \rightarrow 2 \rightarrow 3$. The type $\mathrm{R}$ nodeGosper curve in Figure 3(b) can be described as $5 \rightarrow 4 \rightarrow$ $3 \rightarrow 2 \rightarrow 0 \rightarrow 6 \rightarrow 1$, and the path of the type R level-2 node-Gosper curve in Figure 4(a) can be described as follows using the address shown in Figure 5(b):

$$
\begin{aligned}
& 56 \rightarrow 55 \rightarrow 50 \rightarrow 51 \rightarrow 52 \rightarrow 53 \rightarrow 54 \rightarrow 46 \rightarrow \\
& 45 \rightarrow 44 \rightarrow 43 \rightarrow 40 \rightarrow 41 \rightarrow 42 \rightarrow 36 \rightarrow 35 \rightarrow \\
& 34 \rightarrow 33 \rightarrow 30 \rightarrow 31 \rightarrow 32 \rightarrow 24 \rightarrow 23 \rightarrow 22 \rightarrow \\
& 21 \rightarrow 20 \rightarrow 25 \rightarrow 26 \rightarrow 02 \rightarrow 01 \rightarrow 00 \rightarrow 03 \rightarrow \\
& 04 \rightarrow 05 \rightarrow 06 \rightarrow 64 \rightarrow 63 \rightarrow 60 \rightarrow 65 \rightarrow 66 \rightarrow \\
& 61 \rightarrow 62 \rightarrow 16 \rightarrow 15 \rightarrow 14 \rightarrow 13 \rightarrow 10 \rightarrow 11 \rightarrow
\end{aligned}
$$

12.

\section{Related Works}

3.1. Data Collection Methods for WSNs. Generally, the data transmission methods of WSNs can be divided into three categories: direct transmission, multihop transmission, and cluster transmission.

Direct transmission involves each sensor collecting and directly transmitting data to the base station. It is the simplest data transmission method. However, direct transmission is typically restricted by the communication range of sensors and thus may not work for all the sensors in a network. Furthermore, because the power consumption is proportional to the transmission distance, the energy of sensors that are far away from the base station is exhausted faster than that of others.

For multihop transmission, each sensor transmits data to neighboring nodes that are closer to the base station. This routing method can avoid mass energy consumption for the sensors that are far away from the base station. However, because data from all sensors are continuously transmitted to the base station, those that are close to the base station must transfer more information, resulting in the energy of these sensors being exhausted faster than that of others.

Clustering transmission serves as a compromise between the previous two methods. First, the entire sensor network is divided into several clusters according to a specific topological relationship. Each cluster periodically selects its cluster head according to a protocol, and then all the sensors in the cluster transmit their collected data to the respective cluster head. Finally, the cluster head sends the aggregated data to the base station in a direct or multihop manner. Among all cluster transmission methods, the low-energy adoptive cluster hierarchy (LEACH) [10] is the most well-established.

The LEACH protocol divides the nodes of a WSN by distance into a fixed number (typically $5 \%$ of the total number of nodes) of clusters. Each cluster has a cluster head. The protocol consists of two phases, a setup phase and stabilization phase. In the setup phase, the cluster heads are selected uniformly. Subsequently, all cluster heads send advertisement messages to all reachable nodes, each of which may receive more than one message, in which case, each selects the message with the strongest signal and returns a Join message to the cluster head, thereby enabling each cluster head to determine which nodes belong to its cluster. The LEACH performs the time division multiple access (TDMA) scheduling algorithm in each cluster to determine the time slot of each node in which to send data.

In the stabilization phase, time is divided into multiple intervals. The nodes in a cluster use the schedule information obtained during the setup phase to transfer data to the cluster head. The cluster head aggregates all data received and sends them to the base station. In the LEACH protocol, the setup phase and stabilization phase are continuous cycles. Therefore, determining a new cluster head in each cycle is necessary to evenly dispense the energy of the nodes in a cluster. The main drawback of the LEACH is that the size of each cluster is different (because of the random distribution of the cluster head). This can easily lead to uneven energy consumption by the nodes.

Zigbee is the communication protocol currently adopted by most WSNs. Generally, the outdoor transmission distance of typical Zigbee chips (e.g., those of the TI Chipcon CC2420 series) is $10-60 \mathrm{~m}$. However, if the transmission power of Zigbee can be increased, the outdoor transmission distance can be increased to more than $1 \mathrm{~km}$, as achieved by Digi's XBee S2C series [11]. According to the LEACH report, the energy consumption of radio transmission is proportional to the square of its transmission distance or fourth power when the distance is greater than $87.7 \mathrm{~m}$. Therefore, because of the energy consumption of sensors, traditional data transmission methods tend to use multihop short range transmission; however, this type of transmission increases the delay time of data transmission from sensors to the base station.

Recently, many data collection methods for WSNs have begun using robot cars with communication capabilities and GPS functions to travel within the sensor network range according to a planned path and collect information from the sensors. The advantage of this approach is that it conserves the energy of each sensor node; however, its drawback is that it may cause delays in data transfer, especially for sensor networks that require emergency transmissions. Common trajectories include random trajectories, triangle grid scan (TGS), square Hilbert curve, and Z-shaped curves [12]. 
The authors of [13] proposed a data collection method which uses wireless charging technology for a self-controlled car, called SenCar. Their paper determines how to calculate SenCar's best traveling path so that SenCar can charge the maximum number of low-energy sensor nodes while using the minimum latency to collect data from all sensor nodes. To solve this problem, the authors proposed a two-stage algorithm. In the first stage, the highest possible number of sensor nodes, called anchor nodes, that must be charged is determined. SenCar then charges those anchor nodes along the prearranged path. Meanwhile, SenCar also collects data from the sensors near the anchor nodes. The goal is to select the maximum number of anchor nodes. The length of the path used to charge the anchor nodes must be less than a preset threshold. In the second stage, the effectiveness of data collection is considered and the optimal data transmission rate, scheduling, and path are calculated.

Recent data collection methods for RWSNs such as those proposed in $[14,15]$ use the same approach as that proposed in [13] of combining wireless charging and data gathering functions by applying these two functions over the same path for the same duration. However, for general WSNs, the durations for charging sensors and collecting data are typically different. The duration for data collection is typically measured in seconds and minutes and is much faster than that of charging the sensors in the network (possibly measured in months or years). Therefore, if we use the two functions being performed together as a prerequisite for selecting the charging path or data collection path, the method of choosing the path requires careful consideration.

3.2. Recharge Paths for RWSNs. In this section, I examine representative studies of RWSNs in terms of recharge plans.

The authors of [16] considered the sensor charging problem of WSNs as a location selection problem and also considered the measurement of charging time for mobile charging stations. The goal of their proposed method was to minimize the total charging time and meet the minimum power requirements of the sensors for maintaining fundamental operations. The authors formulated this problem as a linear programming problem.

To select the charging site, the authors of [16] considered two cases. In the first case, the charging location was limited and the location was known. Thus, the problem could be simplified to calculate the individual sensor charging time problem. The goal was to minimize the total charging time of all the sensors. In the second case, the charging site could be anywhere in the sensing area. The authors first narrowed down the possible area of the charging sites and then calculated the available target charging regions. Finally, the possible combinations of these regions were used to calculate the charge time for all the sensors. The goal was to determine the available regions that minimized the total charge time. The authors' charging method was based on a radio power supply calculation formula from [6], which claimed that the power supply energy is inversely proportional to the square of the distance. This implies that the charge source can charge any number of charged devices without restriction. However, this implication may be contentious in the real world, in which charged devices are typically limited. Therefore, the authors' method may require modification for application in real-world settings.

The authors of [17] proposed a hierarchical mobile charging method where mobile charging devices are divided into two layers. Those in the first layer were called mobile chargers and those in the second layer were called special chargers. Within the charging model, each mobile charger was responsible for charging several sensors and each special charger was responsible for charging several mobile chargers. Therefore, solving the WSN charging problem constituted the question of how to divide the sensors into groups and how to group the mobile chargers. The best grouping results were those for which all mobile chargers and special chargers ensured that all sensors were charged in time. This also ensured maximization of the lifetime, connectivity, and coverage of the sensor network. However, energy loss is always incurred when devices are charged, and thus the suitability of using high-capacity charging equipment to charge secondary charging devices instead of directly charging the sensors is questionable.

In [18], Han et al. focused on the charging paths of the mobile wireless charger and the different effects of these paths on the performance of the RWSN. The authors studied four paths, namely, the SCAN, Hilbert curve, S-Curve (ad), and Z-Curve (Figure 6), all of which are typically used for data collection and sensor localization for mobile sinks in a WSN. Similar to the authors of [16], Han et al. used the wireless power supply formula proposed in [6], in which the power supply energy is inversely proportional to the square of the distance. The authors examined the effects of various charging paths on several crucial factors in a sensor network. These factors were as follows: (1) network lifetime, (2) distribution range of energy provision, (3) number of surviving nodes, (4) average charge delay, and (5) efficiency of mobile charger paths. According to the authors' experimental analysis, the S-Curve exhibited the best performance for all factors except for factor (4).

\section{Proposed Data Collection Method and Recharge Plan}

In traditional WSNs, most of the energy for each sensor is provided by the installed batteries. When a sensor's battery power is exhausted, the sensor is considered invalid. A sensor network is considered invalid if over a certain percentage of sensors are invalid. Therefore, to extend the lifetime of a WSN, many operational protocols such as data collection, node localization, and data dissemination consider minimizing and balancing the energy consumption of sensors as a critical design criterion. However, if all sensor batteries are replaced by batteries that can be recharged before they are depleted, the previous operational protocols or methods for WSNs may require reexamination to enable the energy of whole networks to be used more efficiently instead of distributing their energy expenditure among sensors.

In the present study, I focused on the data collection methods of the RWSN and proposed a new method that uses the energy of the whole RWSN more efficiently. Additionally, 


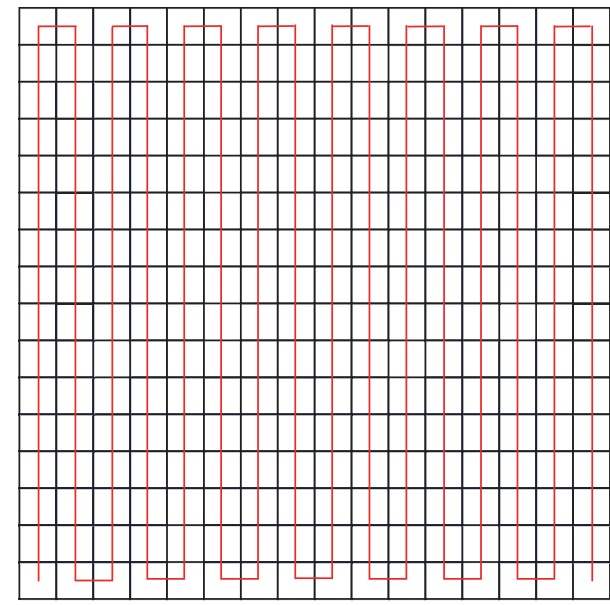

(a)

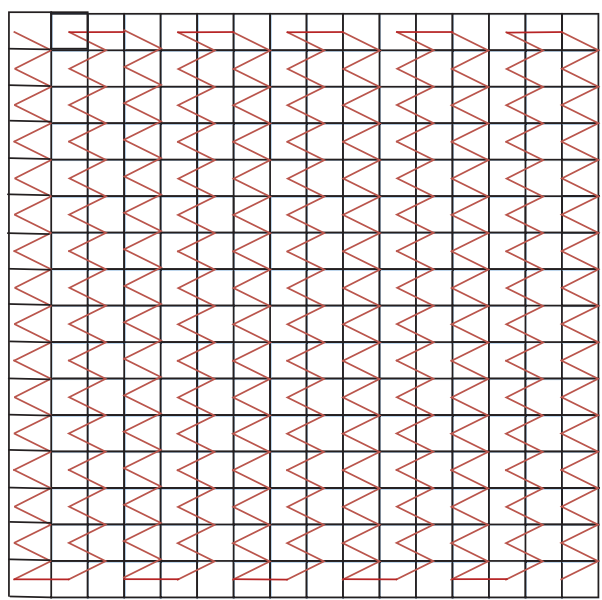

(c)

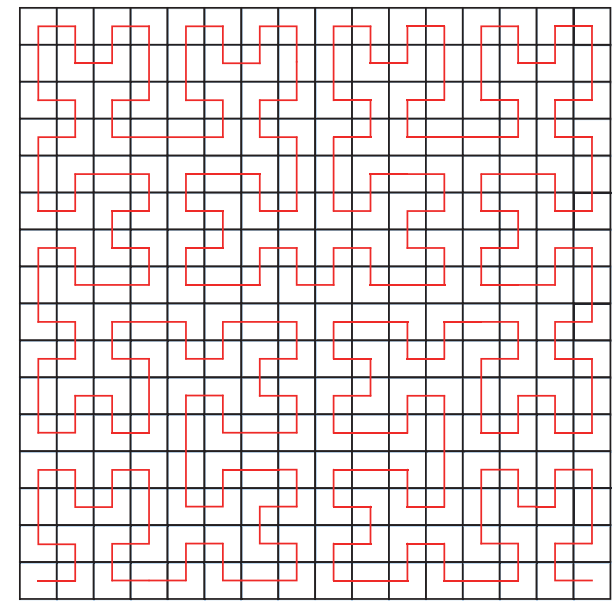

(b)

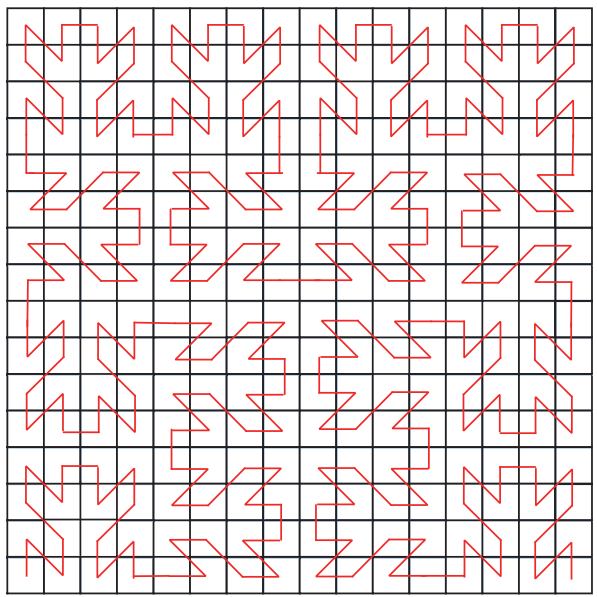

(d)

FIgURE 6: Travel paths of wireless mobile chargers studied in [18]. (a) SCAN, (b) Hilbert curve, (c) S-Curve (ad), and (d) Z-Curve.

in association with the data collection method, I provided an efficient recharge plan to charge all the sensors in the RWSN.

I assumed that the RWSN contains two types of sensors, namely, relay sensors and normal sensors, and that the base station was located at the center of the sensing area. Each relay sensor was installed with the long-range Zigbee communication chip, the communication range of which is over $1 \mathrm{~km}$. Each normal sensor was installed with the normal Zigbee communication chip, the communication range of which is $10-60 \mathrm{~m}$ depending on the environment of the sensing field. Both types of sensors were assumed to have the same amount of initial energy.

4.1. Proposed Data Collection Method for the RWSN. In this subsection, I assume that the entire sensing area of the RWSN was covered by a sufficient level of the node-Gosper island and a relay sensor was deployed at the center of each subnode-Gosper island. In my proposed data collection method, I adopted the hierarchical clustering approach based on the recursive structure of the node-Gosper island. I considered every seven basic hexagons (i.e., the first level node-Gosper island) as a basic cluster. Each sensor transmits its collected data to the center of the central hexagon in its cluster. In order to allow each normal sensor to send its collected data to its cluster head, the radius of the circumscribed circle of each basic hexagon is set to $r / 3$, where $r$ is the communication range of the normal sensors in the RWSN. Because the nodeGosper island has a recursive structure, the higher level nodeGosper island is composed of seven lower level sub-nodeGopser islands. Therefore, the sensor data can be transmitted to the central hexagon of the higher level node-Gosper island using the same method until all the data have been passed to the center of the central hexagon of the entire sensor network.

The proposed data collection method is summarized as the following two stages:

\section{(1) Setup Stage}

(1) Cover the entire sensing area with the minimal level, say $k$, node-Gosper island, in which the radius of the circumscribed circle of each hexagon is set to $r / 3$, where $r$ is the communication range of the normal sensors in the RWSN.

(2) Assign a base-7 address to each hexagon in the level$k$ node-Gosper island and calculate the Cartesian 


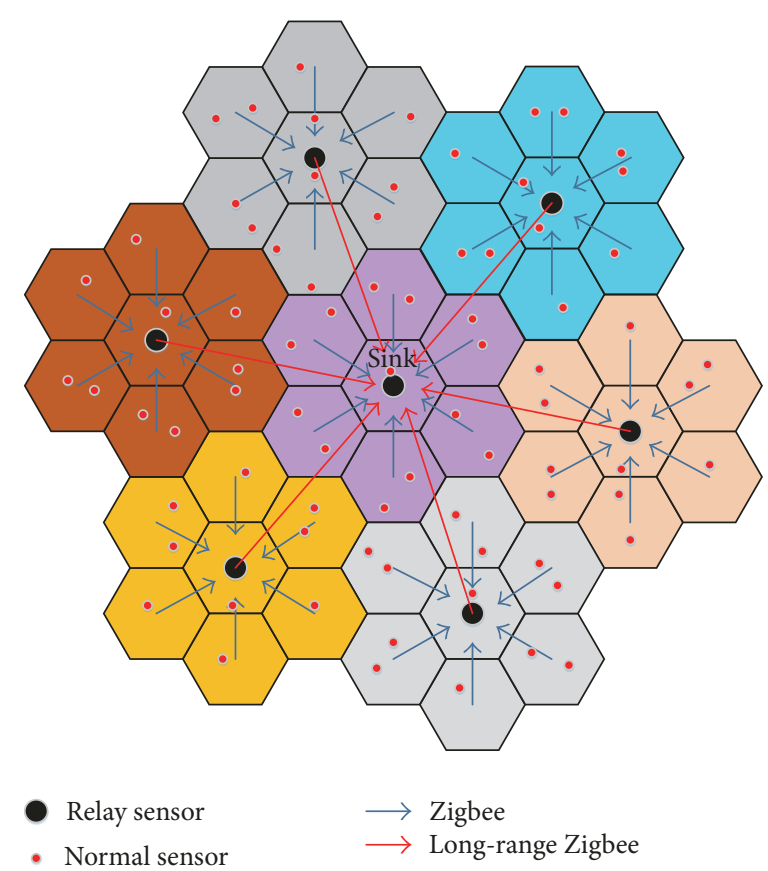

FIGURE 7: Data collection paths in the level-2 node-Gosper island.

coordinates of the center of each addressed hexagon by Theorem 1. Derive the node-Gosper curve of the level- $k$ node-Gosper island by using the base-7 addressing sequence presented in Section 2.2.

(3) Localize all the sensors and separately identify the hexagons that they belong to. This can be achieved by programming a robot car equipped with a wireless communication chip and a GPS device to travel along the level- $k$ node-Gosper curve obtained in Step (2). When the robot car reaches a turning point, it broadcasts its position message to all sensors within its communication range. Once a sensor has received more than three different position messages from the robot car, that sensor can estimate its coordinates by using the trilateration method and subsequently identify the hexagon that the sensor belongs to based on the estimated coordinates.

(4) A relay sensor is deployed at the center of each level-1 sub-node-Gosper island in the level- $k$ node-Gosper island, either manually or by using a robot car (an example of a deployed level-2 node-Gosper island is shown in Figure 7, where the big black points represent relay sensors).

(2) Data Transmission Stage

(1) Each normal sensor sends its data to the relay sensor located at the center of the level-1 sub-node-Gosper island that the normal sensor belongs to based on the address of the hexagon it located.

(2) Each relay sensor in the level-1 sub-node-Gosper island aggregates the collected data and sends the aggregated data to the relay sensor of the level-2 subnode-Gosper island that it belongs to. Similarly, if applicable, each relay sensor of a lower level subnode-Gosper island sends its collected data to the relay sensor of its immediate higher level sub-nodeGosper island until the base station located at the center of the level- $k$ node-Gosper island is reached (Figures 7 and 8 depict the data collection paths of the level-2 and level-3 node-Gosper islands, respectively).

If the aforementioned method is used to transfer the collected data to the base station, the relay sensors of the higher level sub-node-Gosper islands must send more information with longer transmission distances, causing their power consumption levels to increase to levels higher than those of the lower level relay sensors. This phenomenon is restricted by the traditional data collection method in a WSN because the relay sensors of higher level sub-node-Gosper islands become invalid much earlier than other sensors.

In the next subsection, I consider the aforementioned phenomenon and provide details of charging cycles for different sensors with different levels of power consumption to ensure that the power supply to all sensors in the sensor networks is sufficient and stable.

4.2. Recharge Plan for the Sensors in the RWSN. In this study, I outline the use of a wireless mobile charger to charge the sensors in an RWSN. There were two types of chargers assumed to be used in the study. The first type of charger, called M-ChargerCar, was used to charge the relay sensors, which consumed the major portion of the energy in the network. The M-ChargerCar was a robot car equipped with a high-capacity battery, magnetic resonance power transmitter (Figure 2), wireless communication transceiver, and GPS. The second type of charger, called RF-ChargerCar, was used to charge the normal sensors, which consumed a little amount 


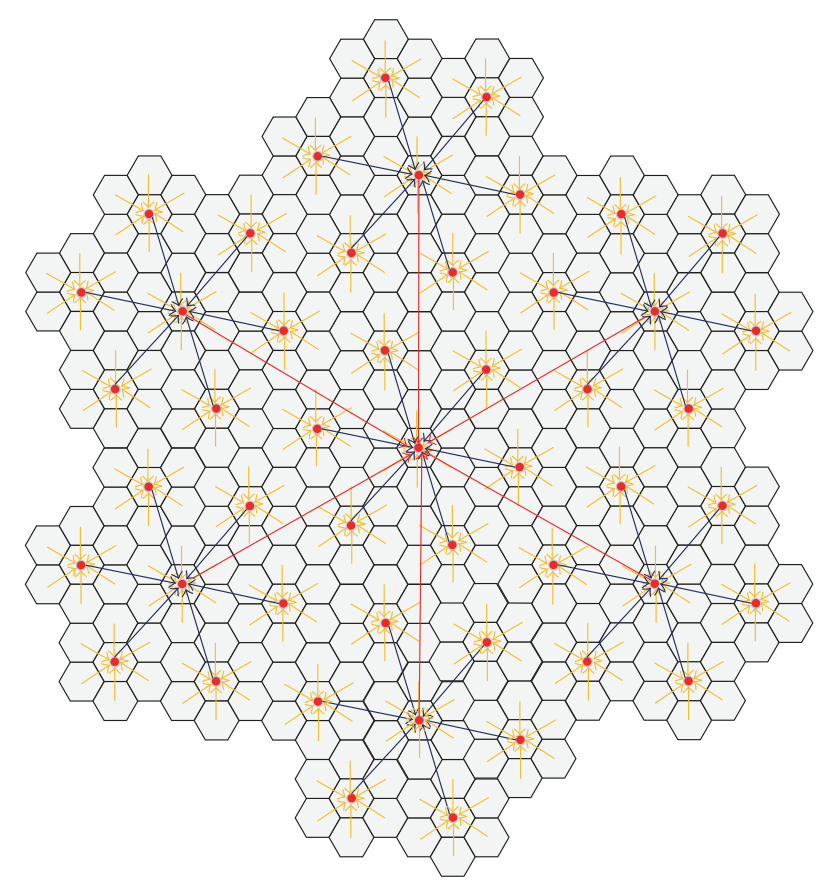

- Relay sensor

FIGURE 8: Data collection paths in the level-3 node-Gosper island (base station is located at the center of the level-3 node-Gosper island.).

of energy because all of them only needed to transmit data within a very short distance. The configuration of RFChargerCar was similar to the M-ChargerCar except that it was equipped with low power radio frequency power transmitter (e.g., TX91503 PowerSpot ${ }^{\circledR}$ Transmitter from Powercast Corporation [19], which can transmit $3 \mathrm{~W}$ power to multiple RF power receivers over $10 \mathrm{~m}$ ).

According to the data collection method proposed in the previous subsection, I assumed that the sensing region of the RWSN is covered by a level- $k$ node-Gosper island. The most power-consuming nodes are the relay sensors located at the center of the surrounding six level- $(k-1)$ sub-nodeGosper islands, which I call layer-1 relay sensors. Considering the level-3 node-Gosper island in Figure 8 as an example, the most energy-consuming nodes are the six layer-1 relay sensors located at the centers of the six level-2 sub-nodeGosper islands. Because I assumed that all the sensors in the RWSN have the same initial energy, the most energyconsuming sensors should be recharged earlier than the other sensors. For this purpose, we can use the path of the level-1 node-Gosper curve depicted in Figure 9 as the routing path for M-ChargerCar to charge the layer-1 relay sensors located at the centers of the level-2 sub-node-Gosper islands.

Subsequently, the relay sensors in each level- $(k-2)$ sub-node-Gosper island called layer-2 relay sensors can be arranged as the next charging nodes by using the level-2 node-Gosper curve. Figure 10 illustrates the level-3 nodeGosper island and corresponding level-2 sub-node-Gosper curve as the charge path for layer-2 relay sensors. Similarly,

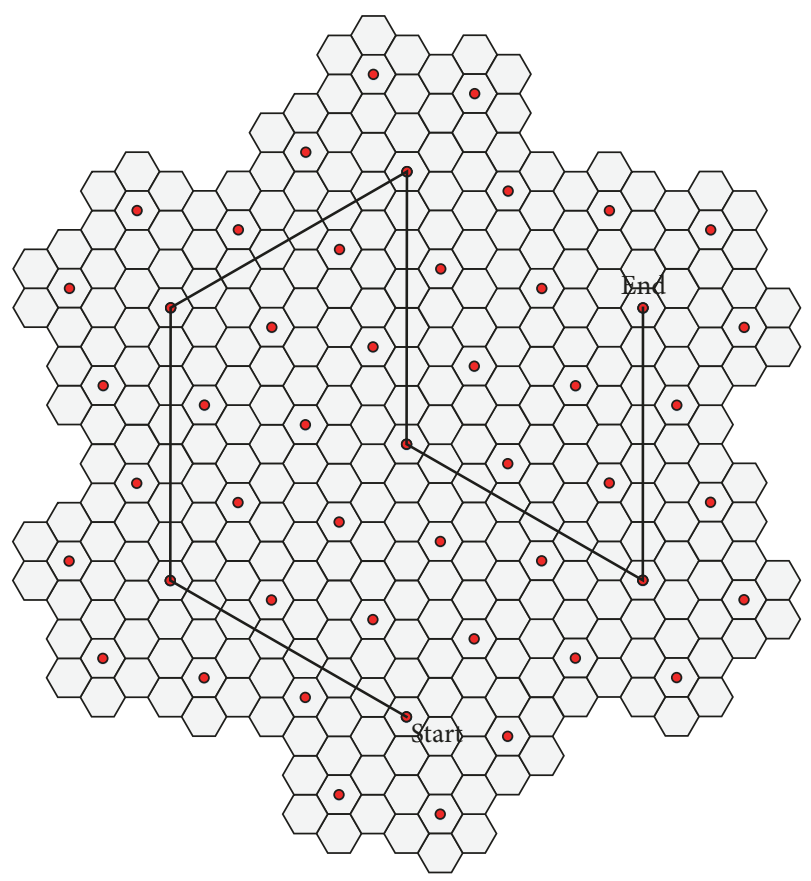

FIGURE 9: Charge path of layer-1 relay sensors: level-1 node-Gosper curve.

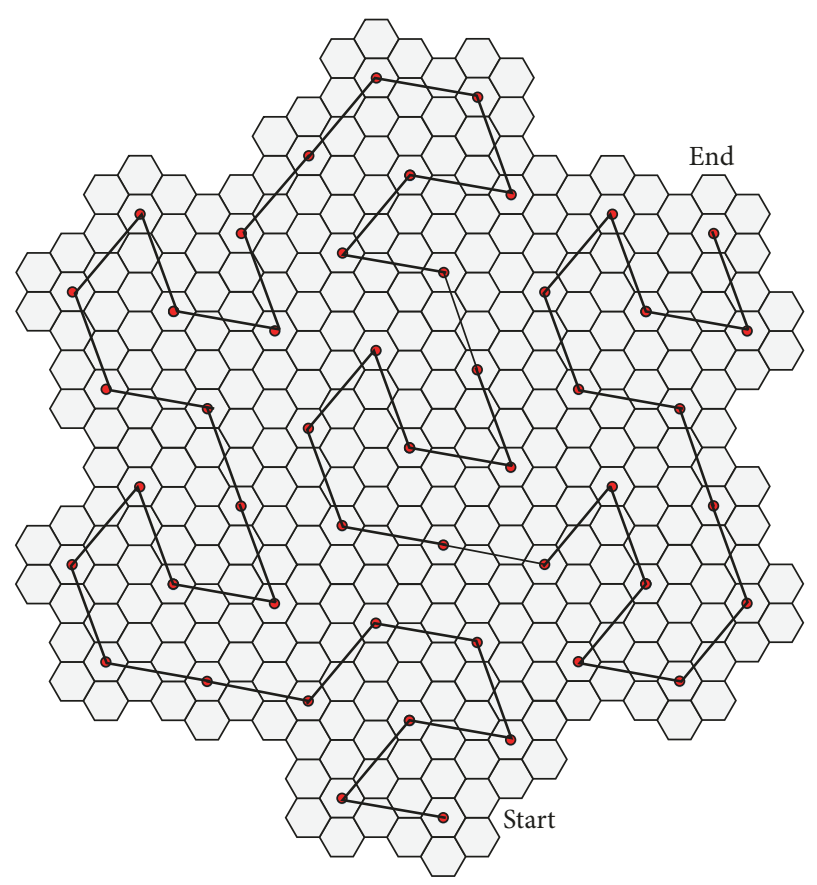

FIGURE 10: Charge path of layer-2 relay sensors: level-2 node-Gosper curve.

for the other relay sensors in level- $(k-i), i=3, \ldots,(k-1)$, subnode-Gosper islands, called layer- $i$ relay sensors, the nodeGosper curve of level- $i$ can be used as the charge path for the relay sensors.

Finally, the charging path for all the normal sensors in the RWSN can follow the level- $k$ node-Gosper curve. As 


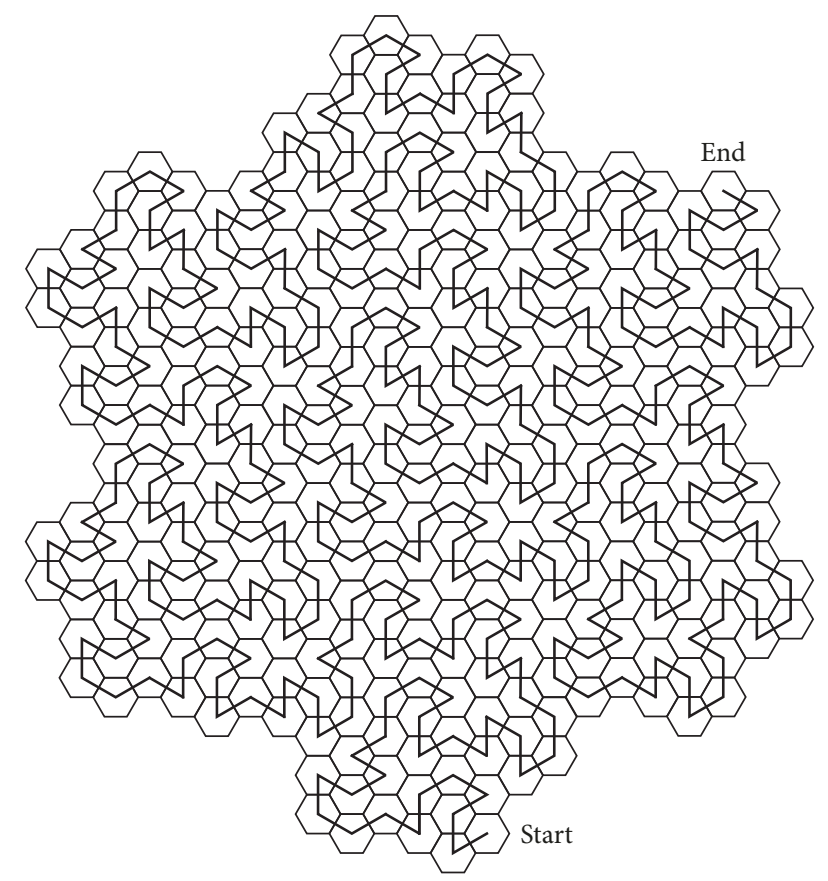

Figure 11: Charge path of normal sensors: level-3 node-Gosper curve.

shown in Figure 11, RF-ChargerCar can stay at the center of each hexagon to charge the sensors in a one-to-many manner in the hexagon. Because each normal sensor in the data collection method mentioned in the previous subsection transmits the sensing data directly and over a short distance to the relay sensor at the center of the level-1 sub-nodeGosper island to which it belongs, the power consumption is very low. Therefore, the energy of each normal sensor can last for an extended period before needing to be recharged.

If the sensing area is covered by a level- $k$ node-Gosper island, the charge plan for the RWSN can be arranged as follows:

(1) Estimate the cycles of recharge time for the relay sensors on each layer and the normal sensors according to the amount of data they have transmitted and received. Suppose that the cycles of recharge time for the relay sensors on layer- $i$ are $R_{i}$ days, where $i=$ $1,2, \ldots, k-1$, and for the normal sensors is $N$ days.

(2) For each $i$ from 1 to $k-1$, identify the centers of the $7^{i}$ level- $(k-i)$ sub-node-Gosper islands that constitute the level- $k$ node-Gosper island. Every $R_{i}$ days, send $\mathrm{M}$-ChargerCar to visit and charge the layer- $i$ sensors located at the $7^{i}$ center positions along the path of the level-i node-Gosper curve.

(3) Every $N$ days, send RF-ChargerCar to travel along the level- $k$ node-Gosper curve and stop at the center of each hexagon (i.e., the turning point of the nodeGosper curve) to charge the normal sensors in the hexagon in the one-to-many manner.

The following text provides an example of estimating the cycles of recharge times for the relay sensors on each layer and that of the normal sensors. Suppose the sensing area is covered by a level-3 node-Gosper island with a radius of $10 \mathrm{~m}$ of the circumscribed circle of each basic hexagon and each sensor is equipped with a $1000 \mathrm{mAh} / 3 \mathrm{~V}$ rechargeable battery with a self-discharging ratio of $10 \%$ per month. Thus, the initial energy for each sensor is $1000 \times 10^{-3} \mathrm{~A} \times 3600 \mathrm{sec} \times$ $3 \mathrm{~V}=10800 \mathrm{~J}$.

Because the communication cost (including the data aggregation cost) is the main portion of energy consumption for each sensor, the following text neglects other costs of sensors except for those specifically mentioned. I adopt the energy consumption model of communication from the LEACH [10] for 200 sensors [the two energy consumption equations are listed in Section 5.1 as (5) and (6)]. The other parameters of the working environment are listed in Table 3. The communication costs of data collection per round for each class of sensors are listed as follows:

(1) Normal sensor: $0.00021 \mathrm{~J}$ [calculated by (5)]

(2) Layer-2 relay sensor: $0.0018 \mathrm{~J}$ [calculated by (5) and (6) and plus data aggregation cost]

(3) Layer-1 relay sensor: $0.0290 \mathrm{~J}$ [calculated by (5) and (6)]

The ratio of the communication costs of the layer1 relay sensor, layer- 2 relay sensor, and normal sensor is $1: 0.0621: 0.0072$. Thus, a fully charged battery for the layer-1 relay sensor can execute $10800 \div 0.0290 \cong 372414$ rounds of data collection. Assuming that each round of data collection in the RWSN lasts $30 \mathrm{sec}$, then

(1) Layer-1 relay sensors must be recharged at least every $(372414 \times 0.5 \mathrm{~min}) \div(60 \times 24) \cong 129$ days (or 106 days if considering self-discharging of the battery);

(2) Each layer-2 relay sensor's battery can be used for $129 \div 0.0621 \cong 2077$ days without considering self-discharging of the battery. If we consider selfdischarging of the battery, each installed battery would have $7.98 \%$ of its initial energy remaining after 2 years, which denotes that each battery must be recharged at least every 2 years;

(3) Although each normal sensor's battery can be used $129 \div 0.0072 \cong 17917$ days without considering self-discharging of the battery, similar to layer- 2 relay sensor's battery, it must be recharged at least every 2 years if considering self-discharging of the battery.

This section describes how long M-ChargerCar requires to fully recharge an equipped battery of a relay sensor. This can be determined according to the Power Transmitter Unit (PTU) of M-ChargerCar and the Power Receiver Unit (PRU) used for the rechargeable battery.

Supposing we use the WT8800 PTU from WiTricity [20], a magnetic resonance wireless power transmitter can provide up to $30 \mathrm{~W}$ of power delivered to a rechargeable battery with $75 \%$ efficiency in conjunction with RB30-RX (an AirFuel ${ }^{\mathrm{TM}}$ certified PRU) [20]. In this case, full charging of the battery installed in each sensor unit of the RWSN in question can be completed in $(10800 \mathrm{~J} / 30 \mathrm{~W}) / 75 \%=480 \mathrm{~s}=8 \mathrm{~min}$. Thus, fully 
TABLE 2: Time taken to fully charge 200 sensors of each layer of relay sensors in the RWSN by using my proposed recharge plan with WT8800 PTU and RB30-RX PRU.

\begin{tabular}{lccc}
\hline Class of sensors & $\begin{array}{c}\text { Time taken to charge all the } \\
\text { sensors (hours) }\end{array}$ & $\begin{array}{c}\text { Time taken to travel the } \\
\text { charge path (hours) }\end{array}$ & $\begin{array}{c}\text { Total time taken } \\
\text { (hours) }\end{array}$ \\
\hline Layer-1 relay sensors (7 sensors) & 0.93 & 0.40 & 1.33 \\
Layer-2 relay sensors (42 sensors) & 5.60 & 1.39 & 6.99 \\
\hline
\end{tabular}

${ }^{*}$ The speed of ChargerCar is $30 \mathrm{~m} / \mathrm{min}$.

TABLE 3: Simulation configuration parameters.

Items

Sensing regions
Values

(1) Square regions of $300 \mathrm{~m} \times 300 \mathrm{~m}$ for direct transmission, multihop, and LEACH

(2) Level-3 node-Gosper island (radius of circumscribed circle of each basic hexagon is $10 \mathrm{~m})$

\begin{tabular}{lc}
\hline Coordinates of base station & $(0,0)$ \\
\hline Number of sensors & $200,300,500$ \\
\hline Data packet size & $4000 \mathrm{bits}$ \\
\hline Control packet size (for TDMA in LEACH and my proposed method) & $100 \mathrm{bits}$ \\
\hline \begin{tabular}{l}
$E_{\text {elec }}($ energy consumption per bit for transmitting or receiving data) \\
\hline $\begin{array}{l}\varepsilon_{f s} \text { (the parameter of the transmission amplifier when the transmission distance } \\
\left.\text { does not exceed the threshold } d_{0}\right)\end{array}$
\end{tabular} & $50 \mathrm{~nJ} / \mathrm{bit}$ \\
\hline $\begin{array}{l}\varepsilon_{m p}(\text { the parameter of the transmission amplifier when the transmission distance } \\
\left.\text { exceeds the threshold } d_{0}\right)\end{array}$ & $0.0013 \mathrm{pJ} / \mathrm{bit} / \mathrm{m}^{2}$ \\
\hline $\begin{array}{l}E_{\text {DA }}(\text { energy consumption per bit for data aggregation for LEACH and my proposed } \\
\text { method) }\end{array}$ & $5 \mathrm{~nJ} / \mathrm{bit}$ \\
\hline Aggregation ratio (for LEACH and my proposed method) & $60 \%$ \\
\hline Number of rounds & 100 \\
\hline
\end{tabular}

charging all layer-1 relay sensors and all layer-2 relay sensors requires $8 \times 7=56 \mathrm{~min}=0.93 \mathrm{~h}$ and $8 \times 42=336 \mathrm{~min}=5.60 \mathrm{~h}$, respectively.

If the radius of the circumscribed circle of each basic hexagon of the node-Gosper island is $10 \mathrm{~m}$, the path length of the level-1 node-Gosper curve in Figure 9 is $420 \sqrt{3} \mathrm{~m} \cong$ $727.5 \mathrm{~m}$, that of the level-2 node-Gosper curve in Figure 10 is $1440 \sqrt{3} \mathrm{~m} \cong 2494.2 \mathrm{~m}$, and that of the level- 3 node-Gosper curve in Figure 11 is $5923 \mathrm{~m}$. Assuming that the speed of MChargerCar is $30 \mathrm{~m} / \mathrm{min}$, the traveling time of M-ChargerCar for the level-1 node-Gosper curve in Figure 9 is $24.25 \mathrm{~min}=$ $0.40 \mathrm{~h}$ and that for the level-2 node-Gosper curve in Figure 10 is $83.14 \mathrm{~min}=1.39 \mathrm{~h}$.

After summarizing the charge times of relay sensors and M-ChargerCar traveling time, we obtained the following results. To fully charge all the layer-1 relay sensors and all layer- 2 relay sensors requires 1.33 and 6.99 , respectively. These results are summarized in Table 2.

Because the energy of the layer-1 relay sensors lasts for 106 days and charging all the layer-1 sensors only requires $1.33 \mathrm{~h}$, one M-ChargerCar is sufficient to accomplish the job. Similarly, the energies of the layer- 2 relay sensors last for 2 years. Additionally, charging all the layer- 2 relay sensors only requires 6.99. Therefore, one $\mathrm{M}$-ChargerCar is sufficient to complete the recharging task for all layers of relay sensors in the RWSN.

\section{Simulation Results}

5.1. Simulation Environment Setup. In this section, I simulate my proposed data collection method and compare its performance with those of three other well-known data collection methods, namely, direct transmission, the LEACH [10], and multihop routing with minimum hop counts [21]. The setup of the simulation environment is described as follows.

(a) Two types of sensing regions, a level-3 node-Gosper island and square, of approximately the same size, were used in the simulation. The level-3 node-Gosper island with a radius $10 \mathrm{~m}$ of the circumscribed circle of each regular hexagon was used for my proposed method and the square regions of $300 \mathrm{~m} \times 300 \mathrm{~m}$ were used for the other three methods. Because there were 343 basic hexagons in the level-3 node-Gosper island and the circumscribed circle of each basic hexagon was $10 \mathrm{~m}$, the area of the entire node-Gosper island was $343 \times 150 \sqrt{3} \mathrm{~m}^{2} \cong 89114 \mathrm{~m}^{2}$, thereby yielding an approximately square region of $300 \mathrm{~m} \times 300 \mathrm{~m}=$ $90000 \mathrm{~m}^{2}$.

(b) All the sensors were randomly deployed in the sensing region and had same amount of initial energy.

(c) The energy consumption model from the LEACH [10] was applied to all sensors as follows: 


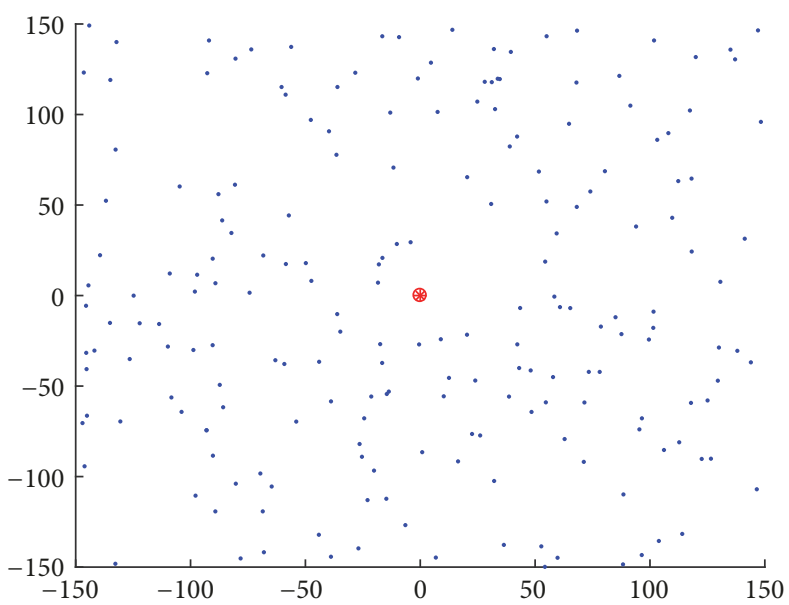

(a) Direct transmission

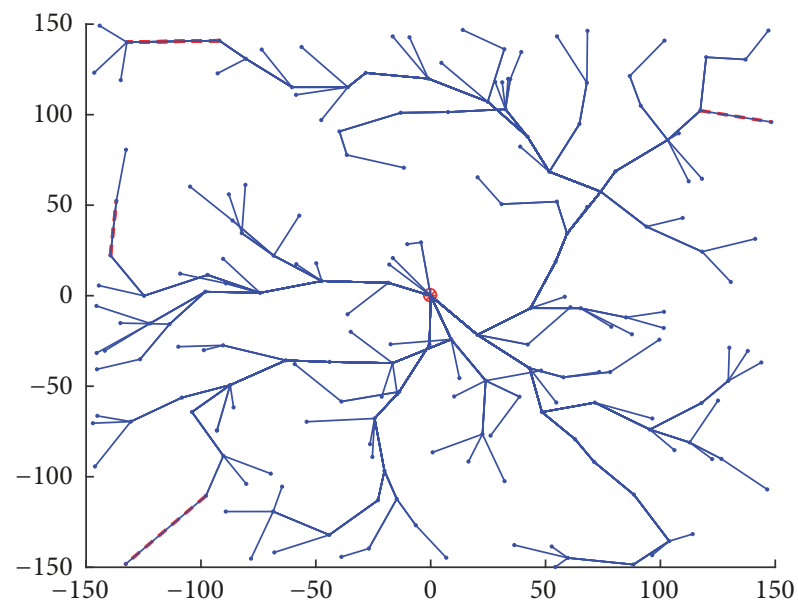

(c) Multihop routing with minimum hop counts (the blue lines are the routing paths and the dashed red lines link the sensors beyond the preset communication distance)

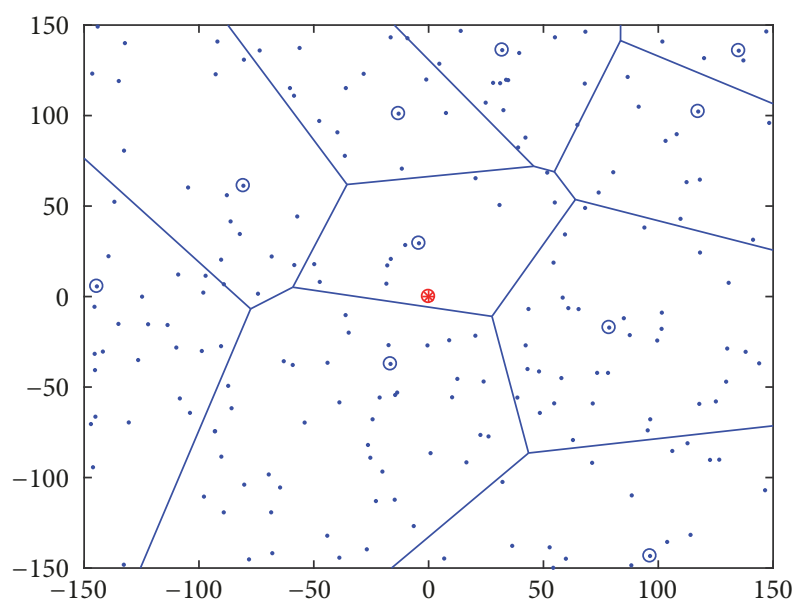

(b) LEACH (the blue double-circled nodes are the cluster heads)

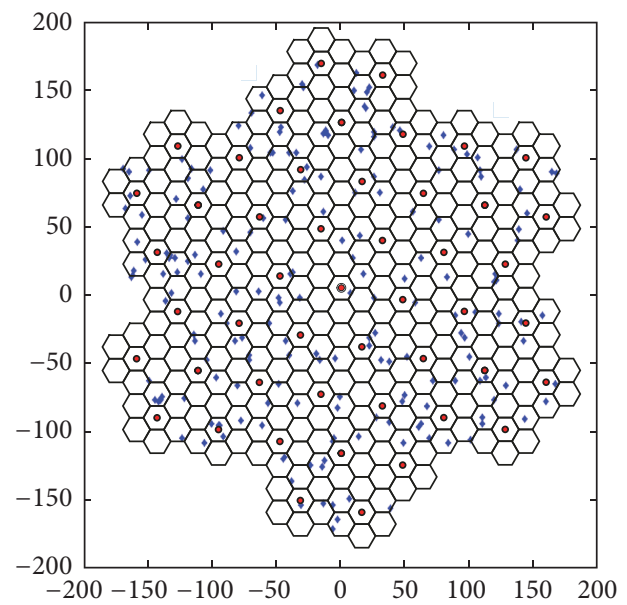

(d) Node-Gosper (my proposed method) (the red points are relay sensors and the blue points are normal sensors)

FIGURE 12: Examples of the distribution of 200 sensors for the four data collection methods (the red double-circled nodes are the base stations located at the center of the sensing area).

$$
\begin{aligned}
E_{\mathrm{TX}}(k, d) & = \begin{cases}k E_{\text {elec }}+k \varepsilon_{f_{s}} d^{2}, & d<d_{0}, \\
k E_{\text {elec }}+k \varepsilon_{m p} d^{4}, & d \geq d_{0},\end{cases} \\
E_{\mathrm{RX}}(k) & =E_{\text {elec }} \times k .
\end{aligned}
$$

Equation (5) is the energy consumption formula for transmitting the packet, where $E_{\mathrm{TX}}$ is the energy consumed when sending packets, $k$ is the size of the packets, $d$ is the distance from the node to the anchor node, $E_{\text {elec }}$ is the energy consumption per bit, $\varepsilon_{f_{s}}$ is the parameter of the transmission amplifier when the transmission distance does not exceed the threshold $d_{0}, \varepsilon_{m p}$ is the parameter of the transmission amplifier when the transmission distance exceeds the threshold $d_{0}$, and $d_{0}=\sqrt{\varepsilon_{f_{s}} / \varepsilon_{m p}}$ is the threshold value of energy consumption. When the value of $d$ is lower than that of the threshold $d_{0}$, the energy consumption is proportional to the square of the distance. When the value of $d$ is greater than that of $d_{0}$, the energy consumption is proportional to the distance from the fourth power. Equation (6) is the energy consumption formula for receiving packets, where $E_{\mathrm{RX}}$ is the energy consumed when receiving packets.

(d) The sensors in the cluster used TDMA as the medium access control layer control protocol for communication.

The details of the configuration parameters of the simulation are listed in Table 3. All the values of the parameter items except the sensing regions were adopted from LEACH in [10] for the sake of consistency and fairness. The value of $d_{0}$ should be $87.7 \mathrm{~m}$ according to the values of parameters $\varepsilon_{f s}$ and $\varepsilon_{m p}$ in Table 3 . The simulation tool used was MATLAB R2013a. Simulations of 500 different deployments of sensors were performed and the mean values were taken for comparison.

Figure 12 illustrates examples of the distribution of 200 sensors in the four data collection methods: direct 


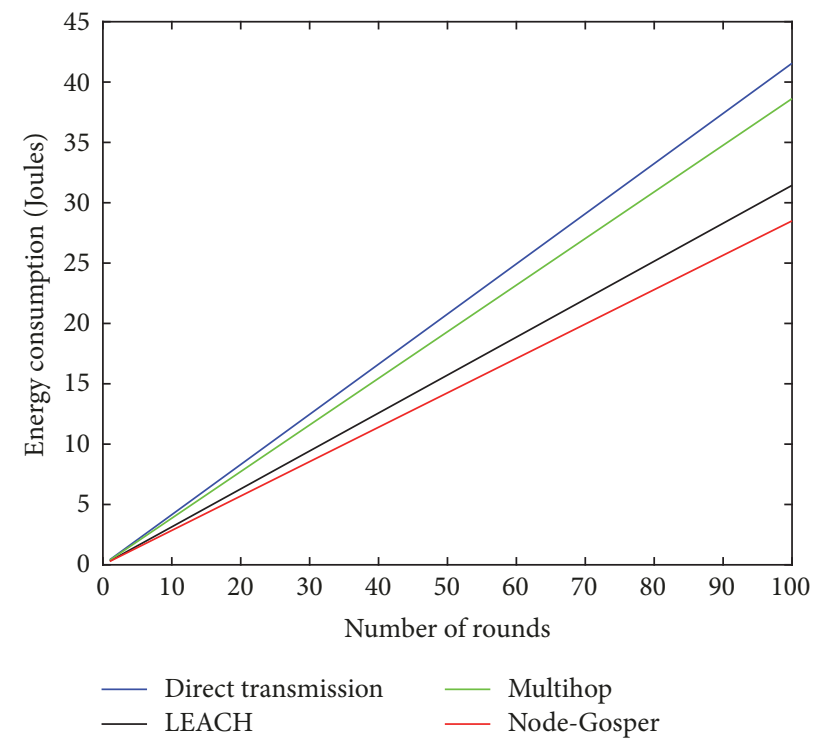

FIGURE 13: Comparison of the accumulated energy consumption of 200 sensors for the four data collection methods.

transmission, the LEACH, multihop routing with minimum hop count, and my proposed method.

\subsection{Comparison of Energy Consumption for Different Data Collection Methods}

(1) Accumulated Energy Consumption of All Sensors. Figure 13 illustrates the simulation results of the accumulated energy consumption for the four data collection methods with 200 sensors. The results are for different numbers of rounds within the environment with the parameters listed in Table 3.

Figure 13 indicates that my proposed method (nodeGosper) is superior to the direct transmission method, multihop routing with minimum hop count method, and LEACH. Figure 12(a) indicates that many sensor nodes are located far away from the base station. According to (5) of the energy consumption model, when the transmission distance is greater than $87.7 \mathrm{~m}$ (i.e., $d_{0}$ ), the transmission energy consumption is proportional to the fourth power of its distance. Therefore, if the direct transmission method is applied, these sensors consume very large amounts of energy and increase the total energy consumption to a very high level.

Regarding the LEACH method, a Voronoi diagram is used for clustering. Because the clusters vary in size, some of the sensor nodes may have communication distances greater than $87.7 \mathrm{~m}$, despite data being sent to their cluster heads within their clusters. Additionally, the election of a cluster head for each round consumes a certain amount of energy from all sensors. These factors render the total energy consumption of the LEACH higher than that of my proposed method.

Regarding multihop routing with minimum hop count method, because sensors do not cluster, no data aggregation effect occurs. Although the distance between each hop is

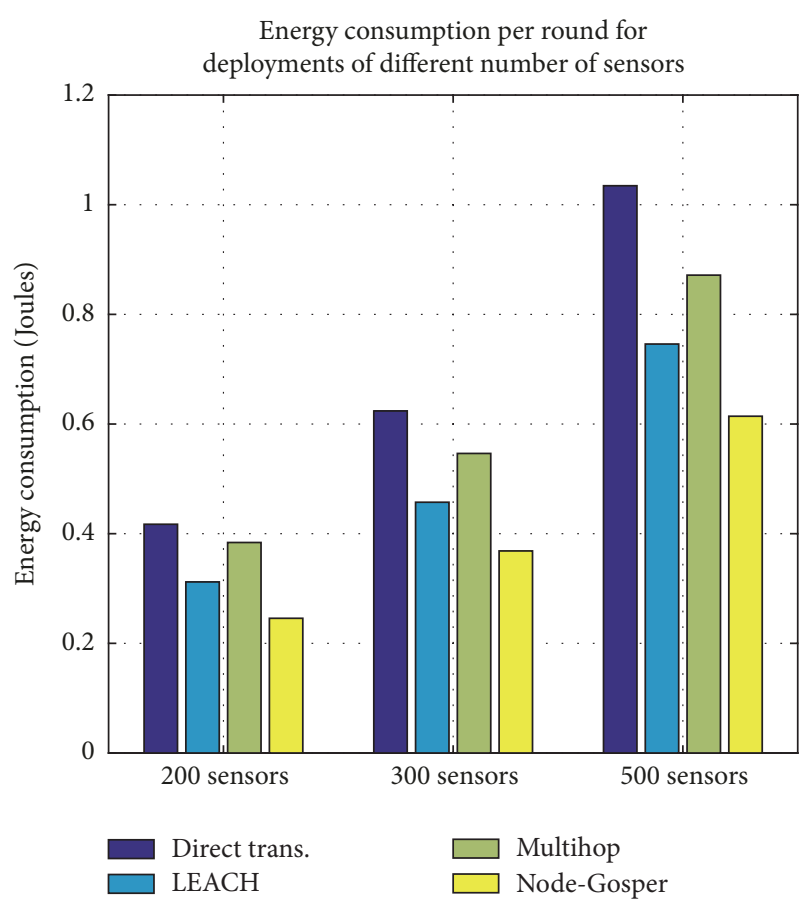

FIGURE 14: Comparison of the average energy consumption per round for different number of sensors deployed in the sensing area for the four data collation methods.

shorter, the total energy consumption of all sensors is still higher than that of my proposed method.

In my proposed method, the cluster size and the locations of cluster heads are fixed. Except for the lower layer relay sensors, almost all the transmissions between sensors occur over a short distance, and this conserves energy. Because my approach causes the lower layer relay sensors to consume excessive amounts of energy, this approach may not be suitable for traditional WSNs because of the uneven power consumption of sensors. However, in the RWSN, this problem can be resolved by shortening the recharging cycle for the lower layer relay sensors to achieve a sufficient energy provision environment.

(2) Energy Consumption for Different Node-Densities. In this subsection, different number of sensors is deployed in the sensing area of each data collection method. I compared the average energy consumption of the sensors per round for the deployment of 200,300, and 500 sensors in the same area of each method. The simulation result is shown in Figure 14, which indicates that my proposed method (node-Gosper) consumed less energy than the other three data collection methods for all the cases of different number of sensor deployments.

In my proposed method, as long as the topology of nodeGosper area is fixed, the number of relay sensors is fixed regardless of the increase of the number of sensors. Therefore, as the number of sensors increases, the additional sensors are all normal sensors, which transmit sensing data in a very short distance, and they consume very little amount of energy. Due to the benefit of the data aggregation in the cluster head, 
the relay sensors only need to transmit $60 \%$ (the assumption in the simulation) of the data amount to their upper level. Therefore, the amount of energy consumption is less than the direct transmission method and multihop method, which do not have the clustering effect. For the LEACH method, the number of cluster heads $(5 \%$ of the sensors in this experiment) increases as the number of sensors increases. Thus, there may be more cluster heads which have distance greater than $87.7 \mathrm{~m}$, and these cluster heads consume a large amount of energy while they transmit collected data to the base station.

(3) Average Energy Consumption of Each Sensor. In this subsection, I compare the average energy consumption of each sensor in the four data collection methods. In particular, I focus on the comparison of the normal sensor in my proposed method (node-Gosper) with the sensors in the other methods. In the simulation, I measured the average energy consumption of all the sensors. In my proposed method, I measured energy consumption of two types of sensors, namely, relay sensors and normal sensors.

In order to provide reliable confidence intervals for the sample data, the distributions of the sample data for each method were shown in Figure 15. Figure 15 showed that the distributions of the sample data were approximated to the normal distribution. Thus, with 95\% confidence intervals, Figure 16 provided the comparison of average energy consumption of each sensor per round for the four data collection methods.

Figure 16 indicates that the average energy consumption of the normal sensors in my method is much less than that of the sensors in the other methods. The reason is that the normal sensors, which are the majority of the sensors, in my proposed method only need to send their sensing data to their corresponding cluster heads, and these transmissions are all within a short distance. On the other hand, most of the sensors in the multihop method not only need to send their sensing data, but also need to relay data for other sensors. In the LEACH method, although the noncluster head sensors only need to send the sensing data for themselves, they may locate at a considerable distance to their cluster head due to the large size of the clusters.

As explained in the first subsection, the lower layer relay sensors in my proposed method may consume excessive amounts of energy. This problem can be resolved by shortening the recharging cycle for the lower layer relay sensors to achieve a sufficient energy provision environment in the RWSN.

5.3. Comparison of Various Charge Paths in the Recharge Plans. In the RWSN, the mobile charger traveled along the charge path and stopped at each stop station to charge each sensor in the network. A longer charge path denoted that more energy was required to move the mobile charger. Similarly, more stop stations denoted that more attempts to start the charger were required, and thus more energy was spent. Therefore, the lengths and number of stop stations on the charge path were proportional to the energy consumed by the mobile charger traveling along the charge path. In this study, I used the node-Gosper curve, which is based on the hexagonal tessellation of a plane, as the charge path for the mobile charger to charge all sensors in the RWSN.

In [18], Han et al. used four well-known routing paths from traditional WSNs, namely, the SCAN, Hilbert curve, S-Curve (ad), and Z-Curve, as charge paths for the RWSN. Table 3 compares the path lengths and number of stop stations of the node-Gosper curve, SCAN, Hilbert curve, SCurve (ad), and Z-Curve, assuming that each unit regular polygon (square or hexagon) has the same radius $(r=10 \mathrm{~m})$ of the circumscribed cycle.

For the same normalized area $\left(1000 \mathrm{~m}^{2}\right.$ or $\left.\mathrm{k} \times \mathrm{m}^{2}\right)$, the path length of the node-Gosper curve was shorter than those of the other four curves, implying that the use of the nodeGosper curve as the charge path for the mobile charger leads to less energy consumption compared with the use of the other four curves. This comparison result encouraged us to choose the charge path based on the node-Gosper curve.

In the following, the four charge paths, namely, SCAN, Hilbert, S-Curve (ad), and Z-Curve, in [18] were compared with the proposed charge path, node-Gosper curve, in three aspects: (1) alive nodes over time, (2) traveling efficiency, and (3) average charging latency. For fairness of the comparison, the same parameter configurations were used and listed in Table 5, and the formula (1) in Section 2.1 with $1 \mathrm{~W}$ transmit power was used as the wireless power transfer model in the simulations.

Figure 17 shows the alive nodes over time for nodeGosper curve, SCAN, Hilbert, S-Curve (ad), and Z-Curve. The number of nodes was set to 200 , and the nodes were distributed randomly in the sensing field with initial energy $1 \mathrm{~J}$ for each one. A node is defined to be alive if its residual energy is greater than zero. The charging time for each charge stop is $8 \mathrm{~s}$.

As we can see in Figure 17, the number of alive nodes starts to decrease after 10,000 seconds, and the number of alive nodes under node-Gosper curve is always greater than that of the other four charge paths. That is because nodeGosper curve has more charge stops and shorter path length than those of the other paths according to the calculation results in Table 4. Having more charge stops implies that there were more nodes near the stops which can be charged more energy by the charger during the trip, and having shorter path implies that less time was spent on the charge path.

Traveling efficiency is defined as the ratio of the total received energy of all nodes and the time of the mobile charger for traversing over the whole network for one time. Figure 18(a) showed the results of traveling efficiency versus number of nodes with charging time $5 \mathrm{~s}$ at each stop. Figure 18(b) was the results of traveling efficiency versus charging time with 200 nodes. Both figures demonstrated that charging path over node-Gosper curve had better traveling efficiency because the node-Gosper curve has more number of charge stops and shorter length of travel path than that of the other four charge paths.

The average charging latency is defined as the average time since the energy of node drop to a threshold ( $0.8 \mathrm{~J}$ in this experiment) to the time the charger stops to deliver power 


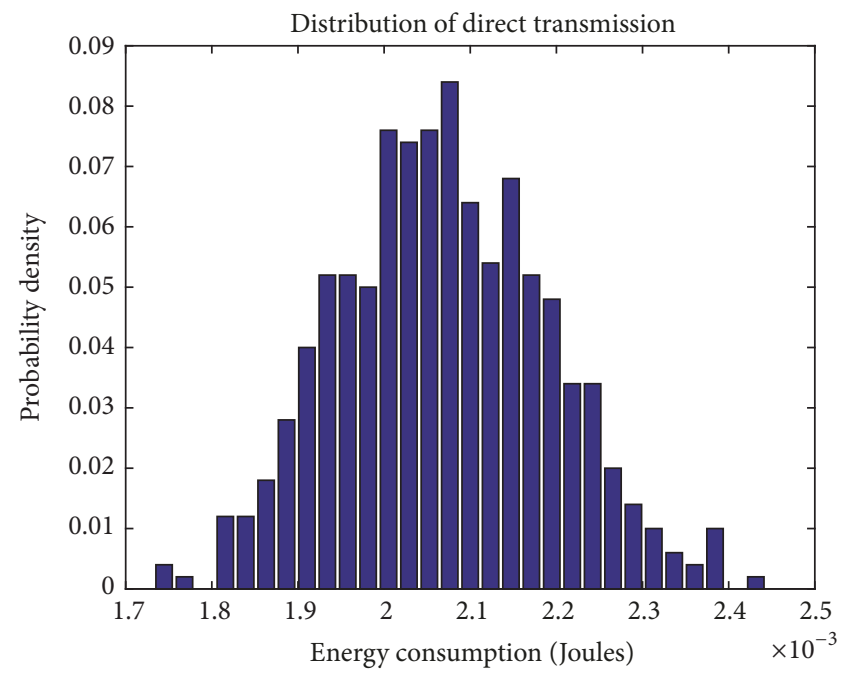

(a)

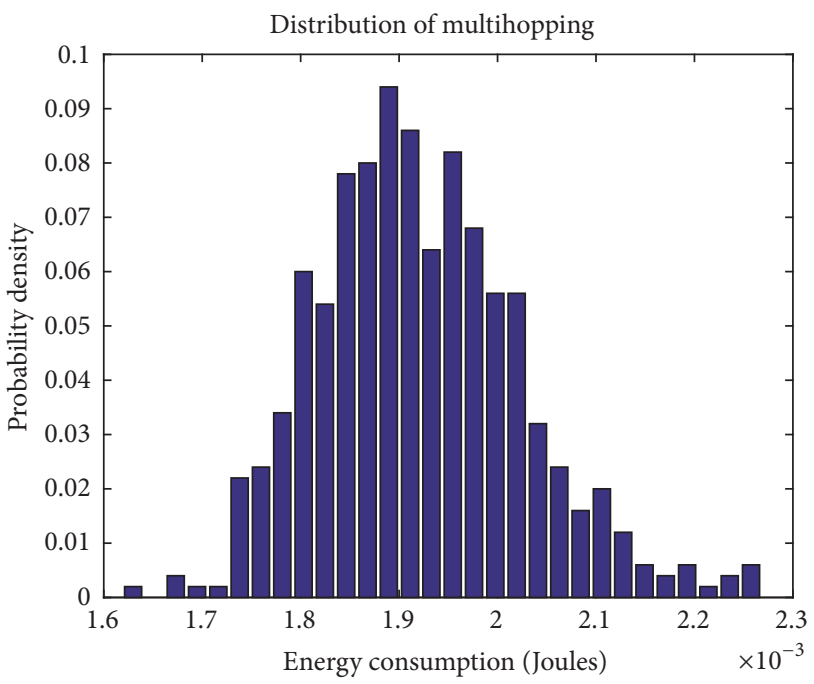

(c)

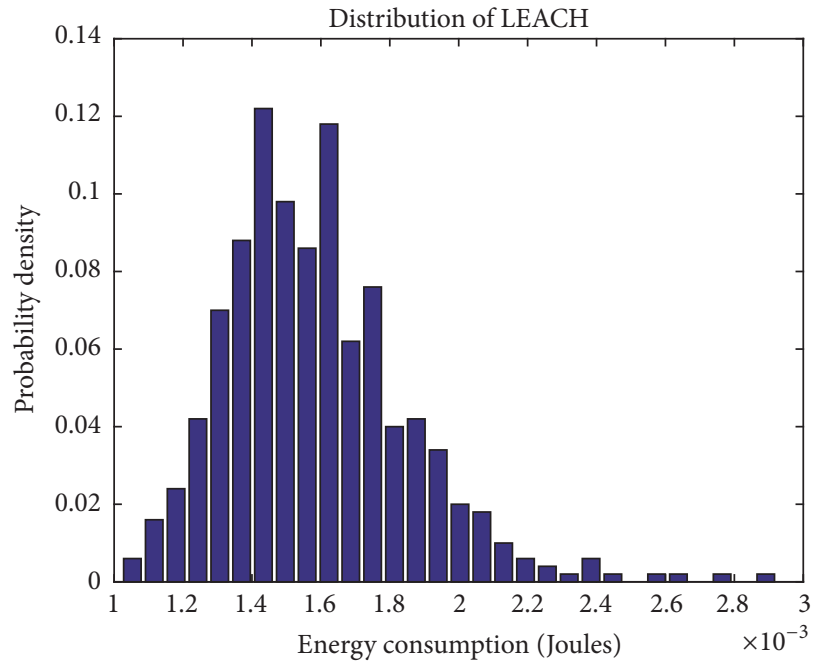

(b)

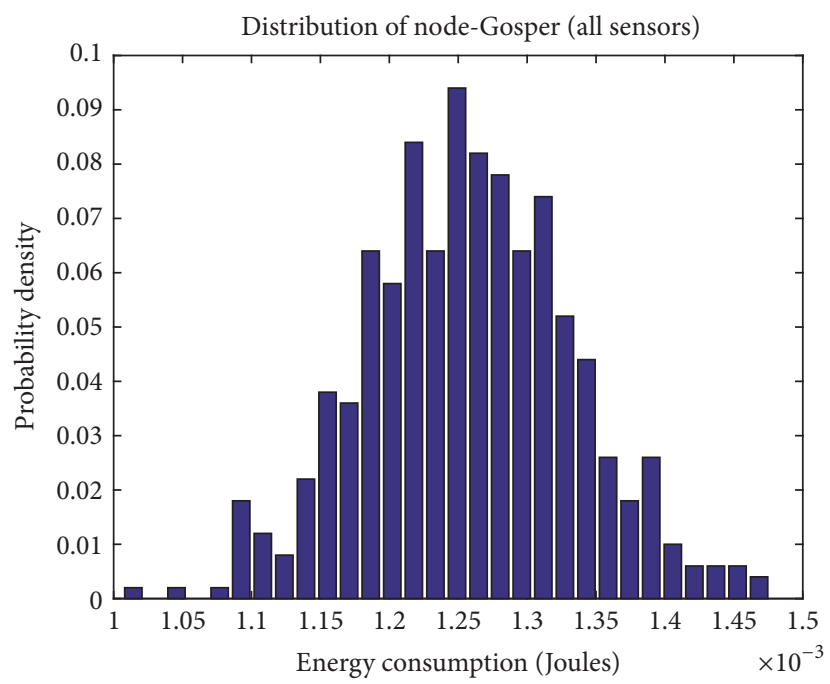

(d)

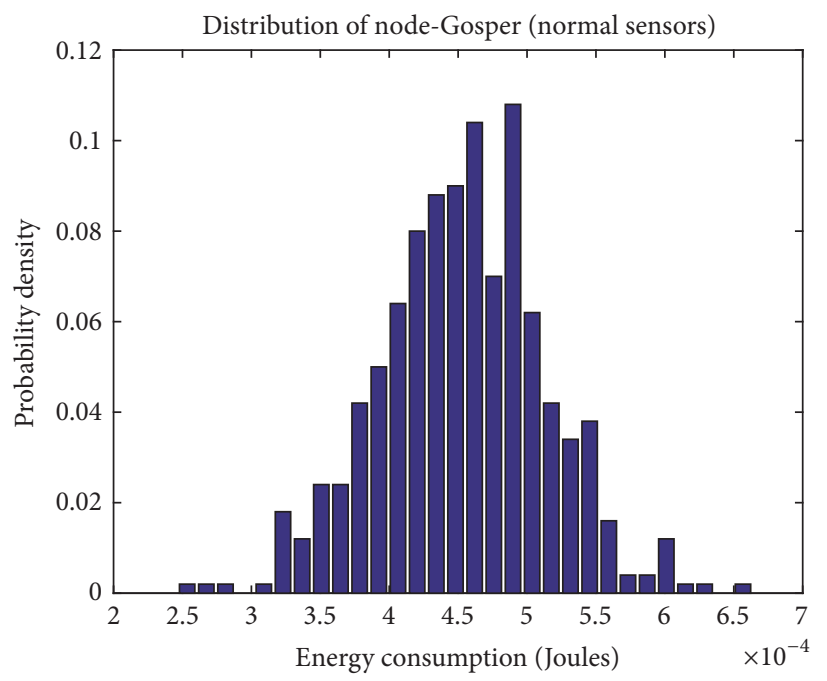

(e)

FIGURE 15: Distributions of average energy consumption of each sensor per round for the four data collection methods (a). Direct transmission (b). Leach (c). Multihopping (d). Node-Gosper (all sensors) (e). Node-Gosper (normal sensors). 
TABLE 4: Path lengths and number of stop stations required to charge all sensors in the RWSN.

\begin{tabular}{lcccc}
\hline Charge path $^{*}$ & Sensing Area $\left(\mathrm{k} \times \mathrm{m}^{2}\right)$ & $\begin{array}{c}\text { Number of stop } \\
\text { stations }\end{array}$ & Path length (m) & ${\text { Path length per } \mathrm{k} \times \mathrm{m}^{2}}^{2}$ \\
\hline SCAN (16 $\times 16$ unit squares) Figure 6(a) & 51.20 & 256 & 3606 & $\mathbf{7 0 . 4 3}$ \\
Hilbert (order 4) Figure 6(b) & 51.20 & 256 & 3606 & $\mathbf{7 0 . 4 3}$ \\
S-Curve (ad) Figure 6(c) & 51.20 & 343 & 3520 & $\mathbf{6 8 . 7 5}$ \\
Z-Curve (order 4) Figure 6(d) & 51.20 & 320 & 4188 & $\mathbf{8 1 . 8 0}$ \\
Node-Gosper curve (level 3) Figure 4(b). & 89.12 & 343 & 5923 & $\mathbf{6 6 . 4 6}$ \\
\hline
\end{tabular}

${ }^{*}$ The radius of the circumscribed circle of each unit square (or hexagon) was $10 \mathrm{~m}$.

TABLE 5: Parameter configurations.

\begin{tabular}{lc}
\hline Parameters & Values \\
\hline Area & $80 \mathrm{~m} \times 80 \mathrm{~m}$ (same area for level-3 node-Gosper) \\
Number of nodes & $160,180,200,220,240,260$ \\
Charger speed & $1 \mathrm{~m} / \mathrm{s}$ \\
Charging range & $5 \mathrm{~m}$ \\
Charging time & $2 \mathrm{~s}, 4 \mathrm{~s}, 6 \mathrm{~s}, 8 \mathrm{~s}, 10 \mathrm{~s}$ \\
Initial energy & $1 \mathrm{~J}$ \\
Energy threshold & $0.8 \mathrm{~J}$ \\
Resolution & $5 \mathrm{~m}$ \\
Energy consumption rate & $0.0001 \mathrm{~J}$ \\
\hline
\end{tabular}

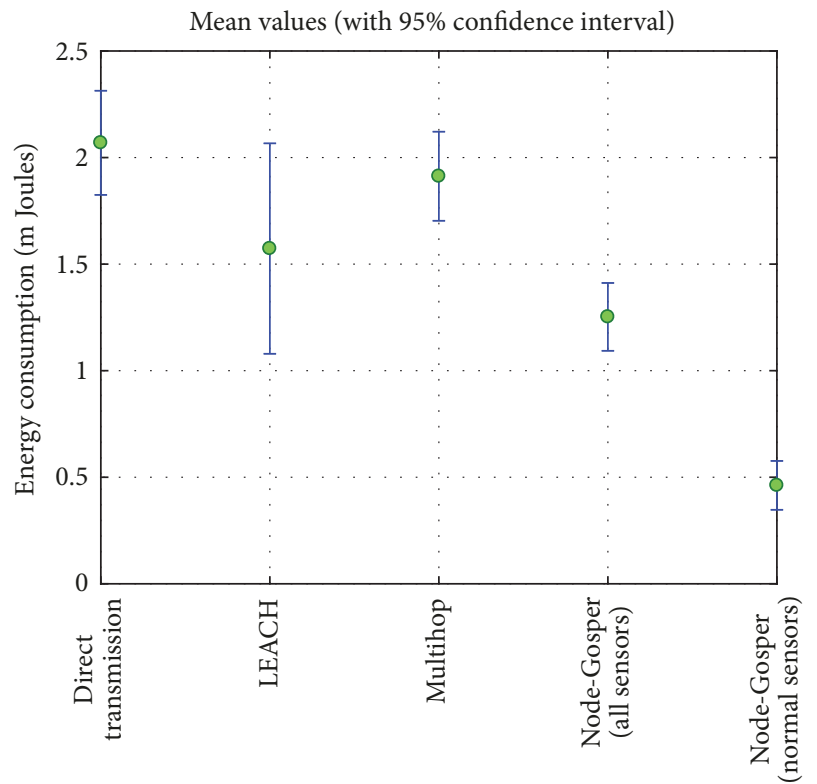

FIGURE 16: Comparison of the average energy consumption of each sensor per round for the four data collection methods.

to it. From Figure 19, we can see that the average charging latency of node-Gosper curve is longer than that of SCAN, Hilbert, and S-Curve and close to that of Z-Curve for the number of nodes from 160 to 220 . The reason is because that node-Gosper curve has more number of charge stops and each charge stop spends $5 \mathrm{~s}$ to charge nodes and thus increases the waiting time for the nodes with energy under the threshold.

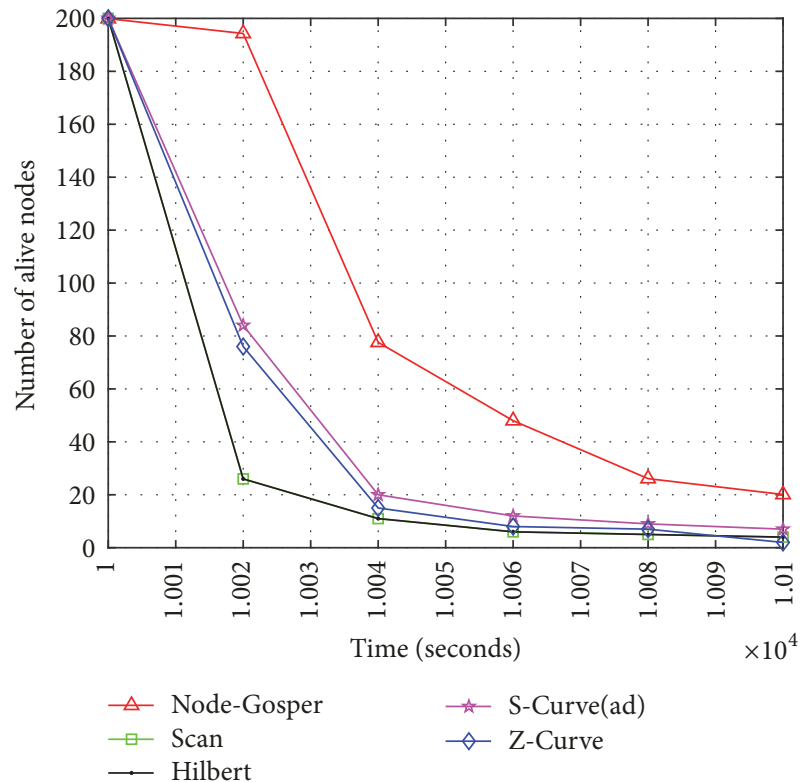

Figure 17: Alive nodes over time for node-Gosper curve, SCAN, Hilbert, S-Curve (ad), and Z-Curve.

\section{Conclusions}

This paper presents a novel data collection method with a recharge plan for RWSNs. My method is based on the nodeGosper island in association with its corresponding nodeGosper curves. I compared my proposed method with three well-known data collection methods, namely, direct transmission, the LEACH, and multihop routing with minimum 


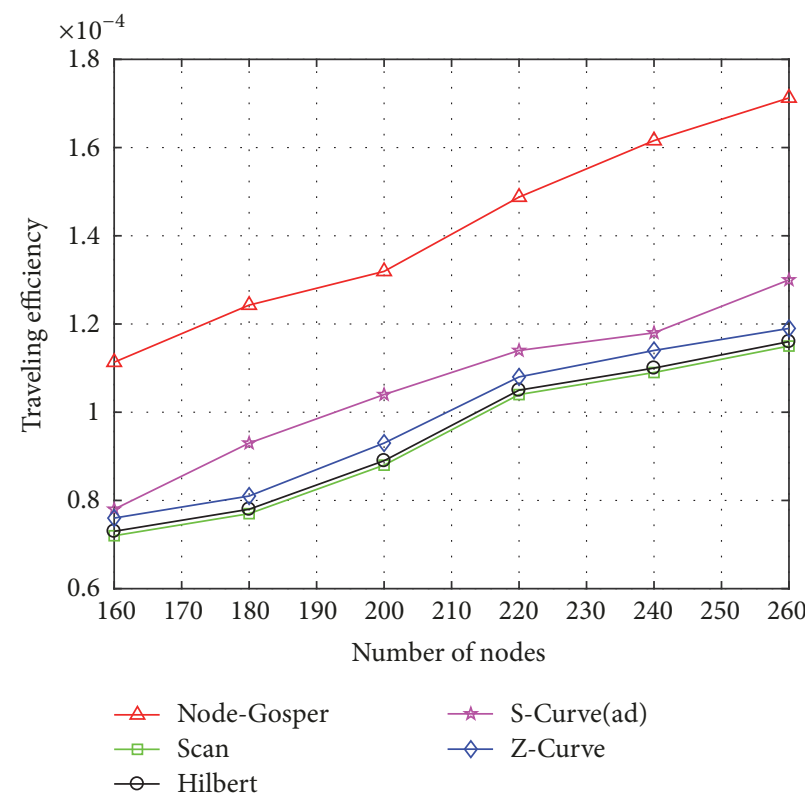

(a)

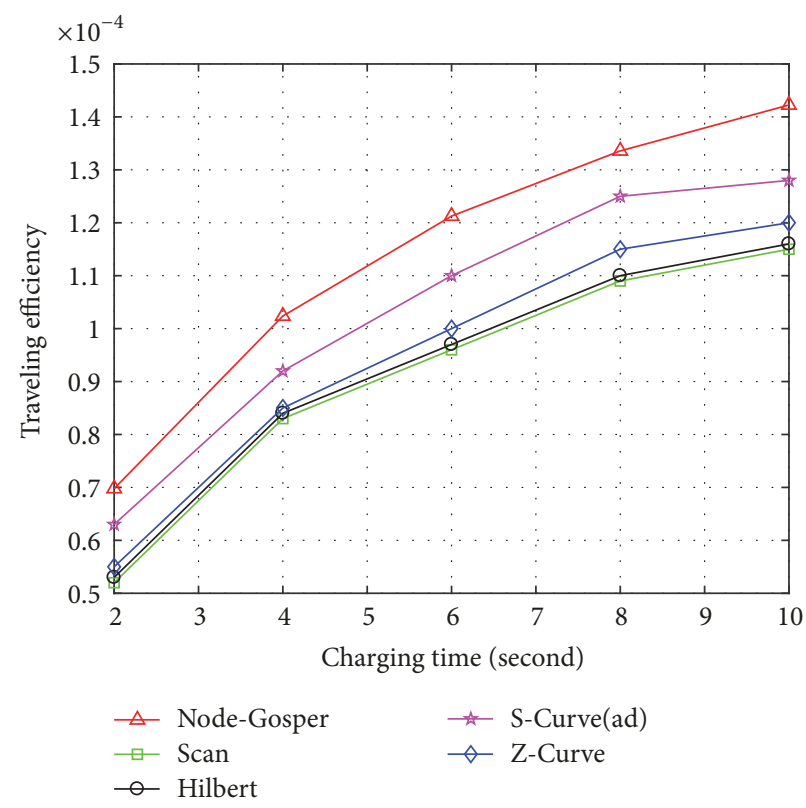

(b)

FIGURE 18: (a) Traveling efficiency versus number of nodes; (b) traveling efficiency versus charging time.

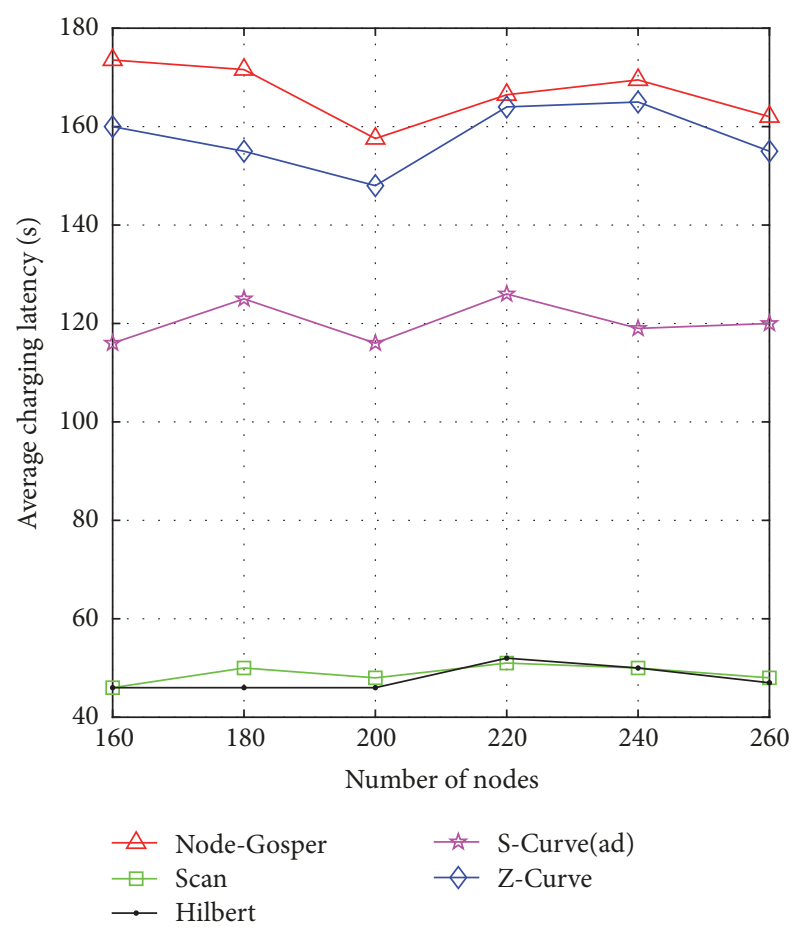

Figure 19: Average charging latency versus number of nodes.

hop count. My proposed method outperformed these three traditional data collection methods in terms of many respects of energy consumption, including the accumulated energy consumption of sensors, different densities of sensors, and average energy consumption of sensors.

I also compared the charge path lengths, alive nodes over time, traveling efficiency, and average charging latency of the charge paths when separately using the node-Gosper curve, SCAN, Hilbert curve, S-Curve (ad), and Z-Curve for the RWSNs. The results in Section 5.3 indicate that the nodeGosper curve had the shortest charge path length, more alive nodes over time, and larger traveling efficiency among all the compared curves. This result implies that the node-Gosper curve is a suitable candidate for use as a charge path for RWSNs.

In recent years, wireless power transfer technologies have increased in popularity in many application domains such as electric vehicles and mobile phones. The cost of wireless power transfer modules is predicted to gradually decrease. Therefore, a rechargeable battery module is expected to soon become available for installation on sensor nodes in WSNs. In such a case, many existing solutions for WSNs from network protocols to working applications must be revisited and adjusted accordingly. I believe that more effort should be devoted to this newly derived research direction in the future.

\section{Conflicts of Interest}

The author declares no conflicts of interest regarding the publication of this paper.

\section{Acknowledgments}

This work was partially supported by the Ministry of Science and Technology, Taiwan, under Contract MOST106-2221-E214-010.

\section{References}

[1] J. Garnica, R. A. Chinga, and J. Lin, "Wireless power transmission: From far field to near field," Proceedings of the IEEE, vol. 101, no. 6, pp. 1321-1331, 2013. 
[2] J. Ventrella, Root 7 Family. Brainfilling Curves-A Fractal Bestiary. Lulu.com, 2012.

[3] C.-C. Chen and S.-B. Wang, "Node-gosper curve-based unknown sensor localization using single mobile anchor in wireless sensor networks," International Journal of Distributed Sensor Networks, vol. 7, p. 13, 2016.

[4] A. Kurs, A. Karalis, R. Moffatt, J. D. Joannopoulos, P. Fisher, and M. Soljačić, "Wireless power transfer via strongly coupled magnetic resonances," Science, vol. 317, no. 5834, pp. 83-86, 2007.

[5] M. Kesler, Highly Resonant Wireless Power Transfer: Safe, Efficient, and over Distance, Witricity Corporation, 2013.

[6] S. He, J. Chen, F. Jiang, D. K. Y. Yau, G. Xing, and Y. Sun, "Energy provisioning in wireless rechargeable sensor networks," IEEE Transactions on Mobile Computing, vol. 12, no. 10, pp. 1931-1942, 2013.

[7] J. N. Bogaid, D. H. Werner, and P. L. Werner, "Optimization of Peano-Gosper fractile arrays for broadband performance using genetic algorithms to eliminate grating lobes during scanning," in Proceedings of the 2005 IEEE Antennas and Propagation Society International Symposium and USNC/URSI Meeting, pp. 755-758, USA, July 2005.

[8] T. G. Spence, D. H. Werner, and J. N. Carvajal, "Modular broadband phased-arrays based on a nonuniform distribution of elements along the peano-gosper space-filling curve," IEEE Transactions on Antennas and Propagation, vol. 58, no. 2, pp. 600-604, 2010.

[9] A. Ehlers, F. Baumann, and B. Rosenhahn, "Boosted fractal integral paths for object detection," Advances in Visual Computing, pp. 458-470, 2014.

[10] W. R. Heinzelman, A. Chandrakasan, and H. Balakrishnan, "Energy-efficient communication protocol for wireless microsensor networks," in Proceedings of the 33rd annual Hawaii international conference on System sciences, pp. 1-10, IEEE, 2000.

[11] Digi International Inc., XBee/XBee-PRO ${ }^{\circledR} \mathrm{S} 2 \mathrm{C}$ ZigBee ${ }^{\circledR} \mathrm{RF}$ Module - Digi International, https://www.digi.com/resources/ documentation/digidocs/pdfs/90002002.pdf.

[12] S. Ghafoor, M. H. Rehmani, S. Cho, and S.-H. Park, "An efficient trajectory design for mobile sink in a wireless sensor network," Computers and Electrical Engineering, vol. 40, no. 7, pp. 20892100, 2014.

[13] M. Zhao, J. Li, and Y. Yang, "A framework of joint mobile energy replenishment and data gathering in wireless rechargeable sensor networks," IEEE Transactions on Mobile Computing, vol. 13, no. 12, pp. 2689-2705, 2014.

[14] S. Guo, C. Wang, and Y. Yang, "Joint mobile data gathering and energy provisioning in wireless rechargeable sensor networks," IEEE Transactions on Mobile Computing, vol. 13, no. 12, pp. 2836-2852, 2014.

[15] B. H. Liu, N. T. Nguyen, V. T. Pham, and Y. X. Lin, "Novel methods for energy charging and data collection in wireless rechargeable sensor networks," International Journal of Communication Systems, 2015.

[16] L. Fu, P. Cheng, Y. Gu, J. Chen, and T. He, "Optimal charging in wireless rechargeable sensor networks," IEEE Transactions on Vehicular Technology, vol. 65, no. 1, pp. 278-291, 2016.

[17] A. Madhja, S. Nikoletseas, and T. P. Raptis, "Hierarchical, collaborative wireless energy transfer in sensor networks with multiple Mobile Chargers," Computer Networks, vol. 97, pp. 98112, 2016.
[18] G. Han, A. Qian, L. Liu, J. Jiang, and C. Zhu, "Impacts of traveling paths on energy provisioning for industrial wireless rechargeable sensor networks," Microprocessors and Microsystems, vol. 39, no. 8, pp. 1271-1278, 2015.

[19] PowerCast Corporation, http://www.powercastco.com/products/ powerspot/.

[20] WiTricity Corporation (2016), LIVE Reference Design - Witricity, http://witricity.com/wp-content/uploads/2016/12/LIVE_Reference_Design_20161229.pdf.

[21] I. F. Akyildiz, W. Su, Y. Sankarasubramaniam, and E. Cayirci, "Wireless sensor networks: a survey," Computer Networks, vol. 38, no. 4, pp. 393-422, 2002. 


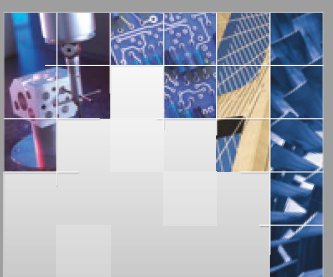

\section{Enfincering}
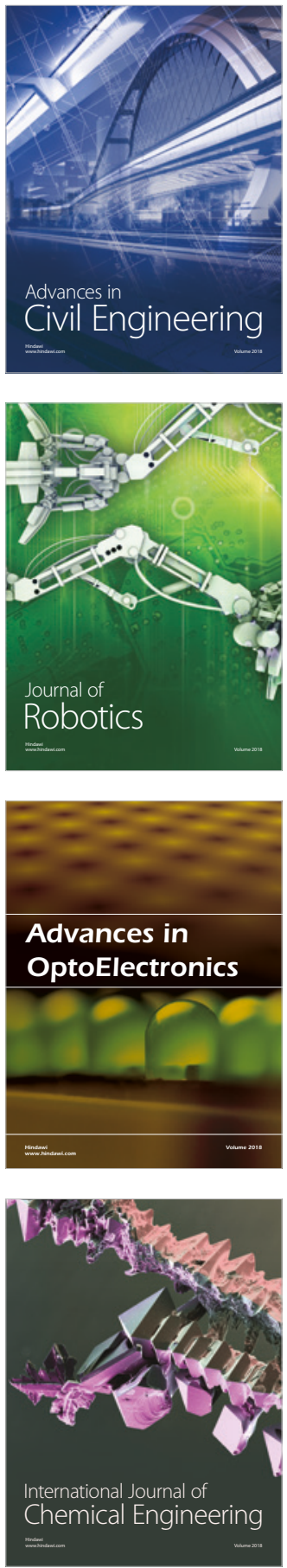

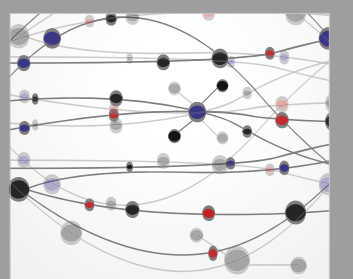

\section{Rotating \\ Machinery}

The Scientific World Journal

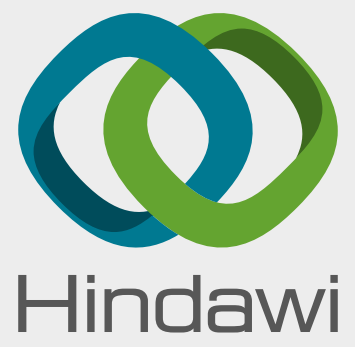

Submit your manuscripts at

www.hindawi.com
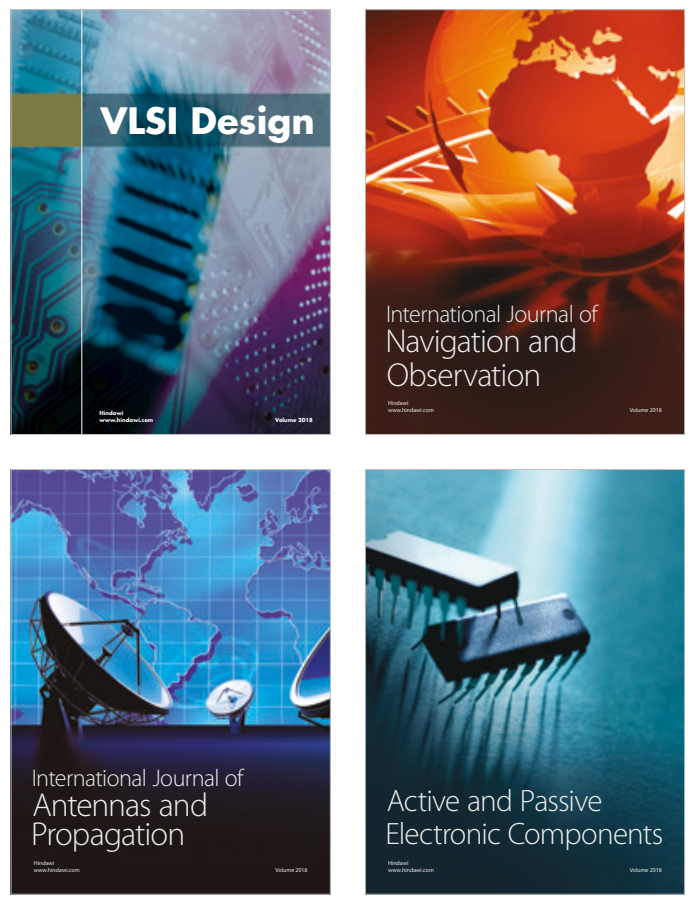
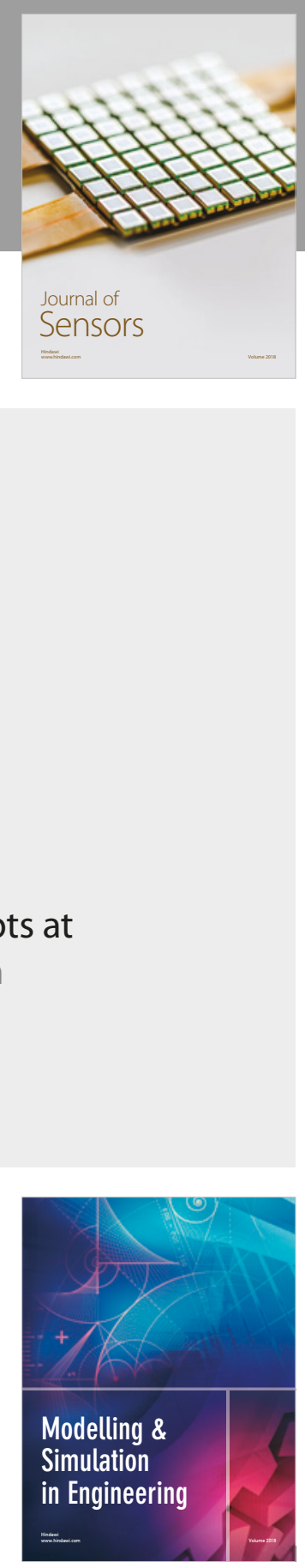

\section{Advances \\ Multimedia}
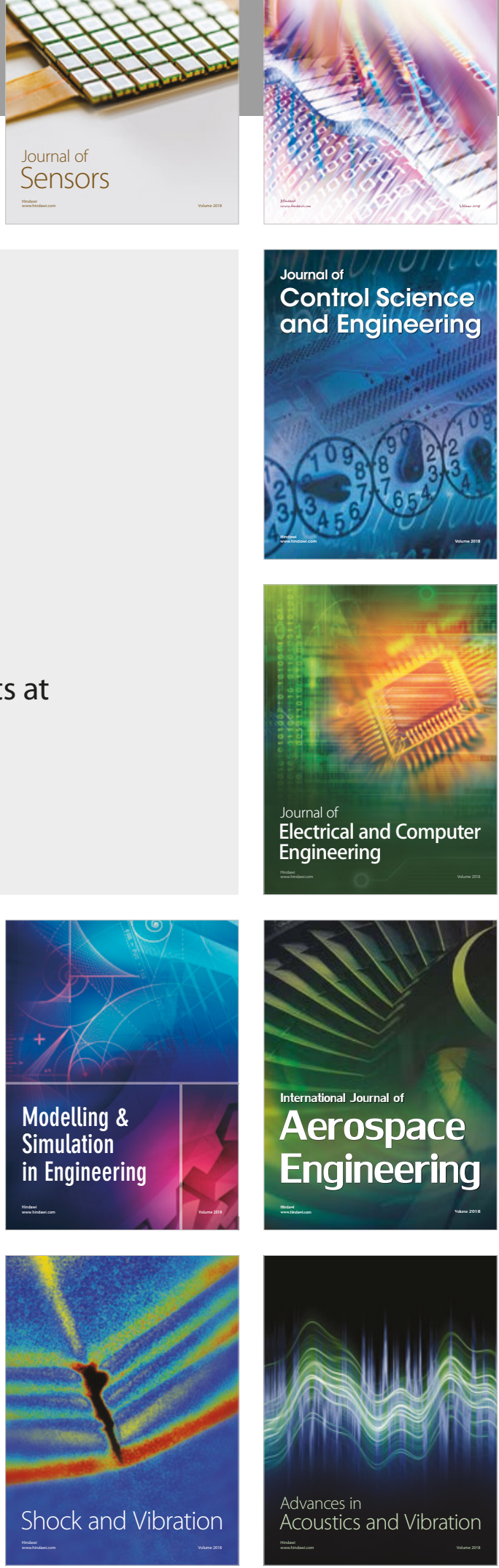